\title{
Plasmonic colorimetric sensors based on etching and growth of noble metal nanoparticles: Strategies and applications
}

\author{
Zhiyang Zhang, ${ }^{\mathrm{a}, \mathrm{d}}$, Han Wang ${ }^{\mathrm{a}, \mathrm{d}}$, Zhaopeng Chen ${ }^{\mathrm{a}, *}$, Xiaoyan Wang ${ }^{\mathrm{b}}$, Jaebum Choo $^{\mathrm{c}, *}$, \\ Lingxin Chen ${ }^{\mathrm{a}, \mathrm{e}, *}$ \\ ${ }^{a}$ Key Laboratory of Coastal Environmental Processes and Ecological Remediation, Yantai Institute of Coastal Zone Research, Chinese Academy of Sciences, Yantai 264003, \\ China

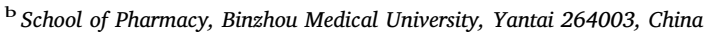 \\ ${ }^{c}$ Department of Bionano Engineering, Hanyang University, Ansan 426-791, South Korea \\ d University of Chinese Academy of Sciences, Beijing 100049, China \\ e College of Chemistry and Chemical Engineering, Yantai University, Yantai 264005, China
}

\section{A R T I C L E I N F O}

\section{Keywords:}

Colorimetric sensors

Plasmonic

Etching

Growth

Metal nanoparticles

\begin{abstract}
A B S T R A C T
Plasmonic colorimetric sensors have emerged as a powerful tool in chemical and biological sensing applications due to the localized surface plasmon resonance (LSPR) extinction in the visible range. Among the plasmonic sensors, the most famous sensing mode is the "aggregation" plasmonic colorimetric sensor which is based on plasmon coupling due to nanoparticle aggregation. Herein, this review focuses on the newly-developing plasmonic colorimetric sensing mode - the etching or the growth of metal nanoparticles induces plasmon changes, namely, "non-aggregation" plasmonic colorimetric sensor. This type of sensors has attracted increasing interest because of their exciting properties of high sensitivity, multi-color changes, and applicability to make a test strip. Of particular interest, the test strip by immobilization of nanoparticles on the substrate can avoid the influence of nanoparticle auto-aggregation and increase the simplicity in storage and use. Although there are many excellent reviews available that describe the advance of plasmonic sensors, limited attention has been paid to the plasmonic colorimetric sensors based on etching or growth of metal nanoparticles. This review highlights recent progress on strategies and application of "non-aggregation" plasmonic colorimetric sensors. We also provide some personal insights into current challenges associated with "non-aggregation" plasmonic colorimetric sensors and propose future research directions.
\end{abstract}

\section{Introduction}

Detection of chemical and biological targets plays a fundamental role in medical diagnosis (Zhou et al., 2015b), environmental monitoring, (Kaushik et al., 2015) and food science (Liu et al., 2010), among other disciplines. Among various analytical methods, colorimetric methods are often favored because of their low cost and simplicity of detection (Saha et al., 2012). Especially, colorimetric methods exhibit great potential in point-of-care testing (POCT) because of their extreme simplicity and low cost since colorimetric assays do not require any expensive or complicated instrumentation. Traditional colorimetric methods based on reactions between organic dyes probes and target analytes are, however, limited by the low extinction coefficients of organic dyes. Therefore, dye-based colorimetric methods are normally not very sensitive.

Nobel metal nanoparticles have increasingly been used for fabrication of colorimetric sensors because of their strong localized surface plasmon resonance (LSPR) extinction in the visible light region (Saha et al., 2012; Zhou et al., 2015b). Due to LSPR, gold nanoparticles possess extremely high extinction coefficients (e.g., $2.7 \times 10^{8} \mathrm{M}^{-1}$ $\mathrm{cm}^{-1}$ for $13 \mathrm{~nm}$ gold nanoparticles), 1000 times higher than those of organic dyes (Ghosh and Pal, 2007), and the detection limit of gold nanoparticle-based colorimetric assays can be in the nanomolar range (even lower range). Metal nanoparticle-based plasmonic colorimetric sensors have been used to detect analytes such as proteins, DNA, anions, metal ions, and organic molecules (Baptista et al., 2008; Saha

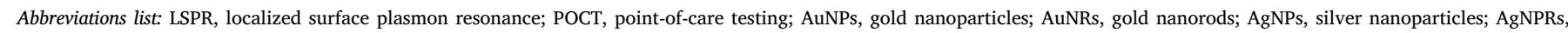

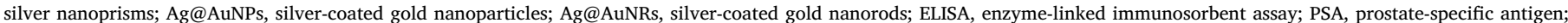

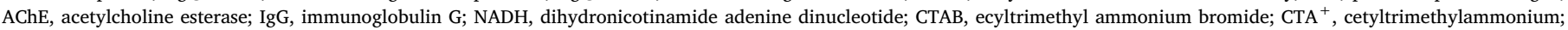
GOx, glucose oxidase; ALP, alkaline phosphatase; SERS, surface enhanced Raman scattering; CD, circular dichroism

* Corresponding authors.

E-mail addresses: zhpchen@yic.ac.cn (Z. Chen), jbchoo@hanyang.ac.kr (J. Choo), lxchen@yic.ac.cn (L. Chen). 

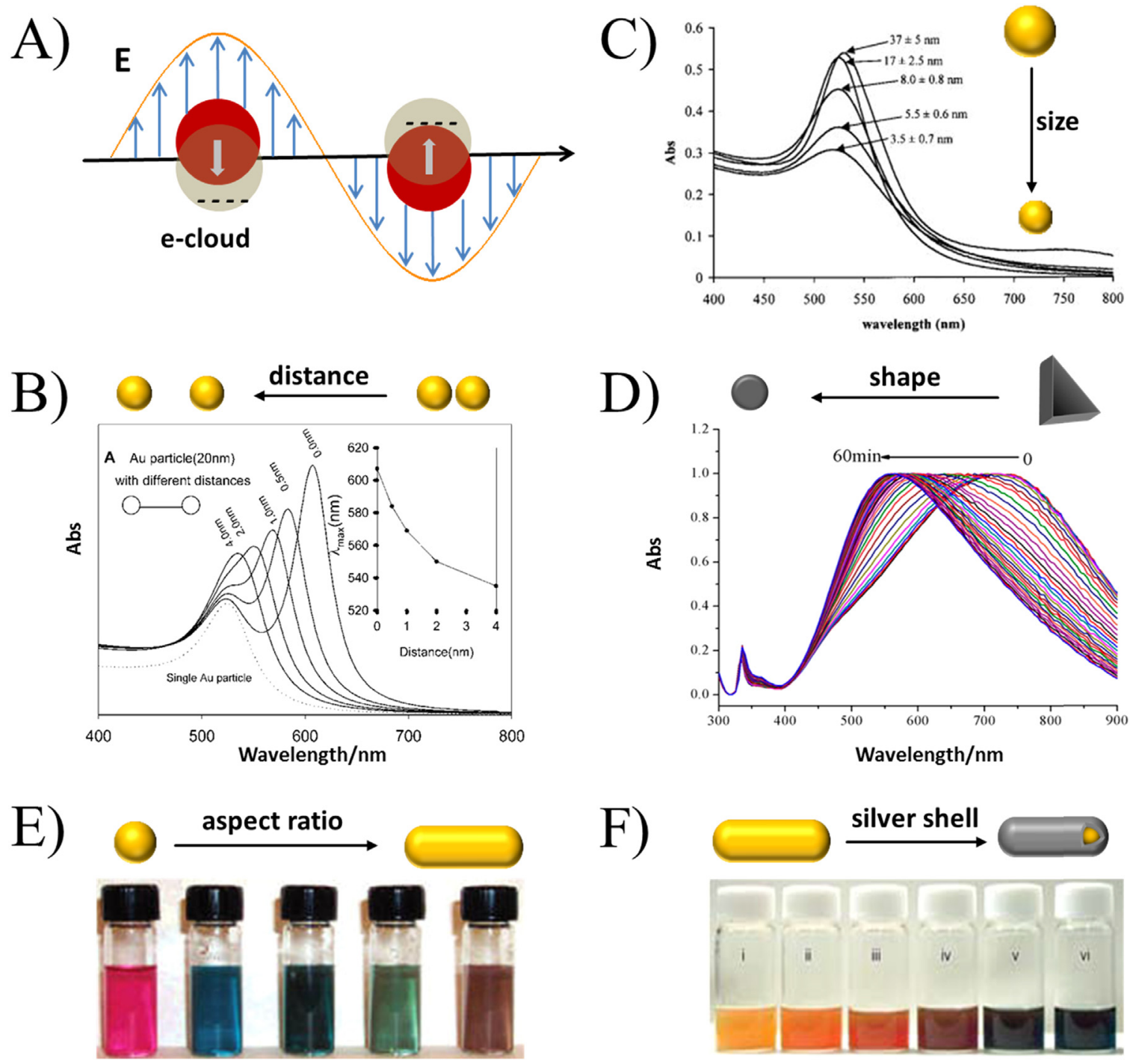

Fig. 1. Dependence of LSPR on nanoparticle size, shape, and composition. (A) Scheme for the LSPR. (B) Electrodynamic modeling calculations for $20 \mathrm{~nm}$ diameter gold nanoparticles with interparticle distance. Inset is the peak shift $v$ interparticle distance. Reprinted (adapted) with permission from Zhong et al. (2004). Copyright: The American Chemical Society. (C) Extinction spectra of different sized gold nanoparticles of 3.5, 5.5, 8.8, 17, and 37 nm, respectively. Reprinted (adapted) with permission from Jana et al. (2001). Copyright: The American Chemical Society. (D) Plasmon peak shift of triangular silver nanoprisms with the shape change induced by the etching of silver nanoprisms in the presence of glucose oxidase and glucose. Reprinted (adapted) with permission from Yang et al. (2014). Copyright: The American Chemical Society. (E) Color of gold nanorods with different aspect ratios. Reprinted (adapted) with permission from Kim et al. (2002)). Copyright: The American Chemical Society. (F) Color of gold nanorods coated with a silver shell of varied thickness. Reprinted (adapted) with permission from Okuno et al. (2010). Copyright: The Royal Society of Chemistry.

et al., 2012; Zhou et al., 2015b).

Among the plasmonic sensors, the most popular mode is the colorimetric sensing approach based on metal nanoparticle aggregation (Elghanian et al., 1997). The principle of these plasmonic sensors is that a decrease in the interparticle distance (induced by the nanoparticle aggregation) leads to a strong plasmon coupling between the nearby particles, causing a redshift in the plasmon bands and an obvious color change of colloidal solution (Ghosh and Pal, 2007). This kind of sensors can be termed as "aggregation" plasmonic colorimetric sensor. As a typical example, the colorimetric detection of DNA can be achieved by inducing the aggregation of gold nanoparticles modified with probe DNA (Elghanian et al., 1997). "Aggregation" plasmonic sensors usually exhibit high sensitivity and simple design, and thus these types of sensors are dominant in the field of plasmonic sensors. However, "aggregation" plasmonic colorimetric sensors usually face two main problems. One problem is the auto-aggregation of colloidal nanoparticles may lead to false positive/negative results, because the nanoparticle aggregation can be caused by many factors, such as solvent, $\mathrm{pH}$, temperature, salt and charged molecules (Ghosh and Pal, 2007). The other problem is the complex modification of reconization reagents (e.g., DNA probes (Lee et al., 2007; Liu and Lu, 2003) and organic ligands (Chen et al., 2011)) on nanoparticles since this procedure usually requires the additional equipment (e.g., High speed centrifuge) and timecomsuming preparation.

In recent years, a so-called "non-aggregation" plasmonic colorimetric sensor becomes a newly-developing research field (Chen et al., 2016a; De La Rica and Stevens, 2012; Yang et al., 2014; Zhang et al., 2017). This kind of sensor is based on the mediation of metal nanoparticles etching and growth which results in the change (damping/ shift) of plasmon band due to the evolution of the size, shape, and dielectric environment of metal nanoparticles (Lee and El-Sayed, 2005), In comparison, "non-aggregation" plasmonic colorimetric sensors have many advantages. Firstly, this kind of sensors has little influence of auto-aggregation of nanoparticles. The used colloidal nanoparticles 
often keep stable in the complex matrix (e.g., sea water) because of the surface stabilizers, such as ecyltrimethyl ammonium bromide (CTAB) (Zhang et al., 2014b). Then, "non-aggregation" plasmonic colorimetric sensors can develop economical and practical test strips by immobilization of nanoparticles on a supporting substrate (Lee and Huang, 2011). The immobilized nanoparticles on test strip not only can decrease the influence of auto-aggregation but also extend their practical utility in POCT due to the simplicity in production and storage. Last not at least, the modification of reconization reagents on metal nanoparticles are not required, which will aviod the complicated modifying processes. Due to their excellent merits, "non-aggregation" plasmonic colorimetric sensor attracts increasing attention and have been applied to various sensing applications, including DNA (Yang et al., 2014), biomarkers (De La Rica and Stevens, 2012), glucose (Zhang et al., 2017), metal ions (Zhang et al., 2014b), and anions (Li et al., 2011) and other targets.

Although plasmonic colorimetric sensors have been reviewed by several researchers (Baptista et al., 2008; Guo et al., 2015; Saha et al., 2012; Tang and Li, 2017; Vilela et al., 2012; Zhao et al., 2006; Zhou et al., 2015a, 2015b), these review articles comprehensively discuss fundamental mechanism, sensing strategies, and applications of plasmonic nanosensors. These reviews have paid more attention to the discussion about "aggregation" plasmonic colorimetric sensors and refractive index-dependent LSPR sensors. It is worth noting that refractive index-dependent LSPR sensors also belong to "non-aggregation" plasmonic sensors (Mayer and Hafner, 2011; Zhao et al., 2006). However, since the band shifts are limited in several nanometers, the optical detector is usually required to mesure the plasmon band shifts and the color change induced by the band shift is hard to differentiate by colorimetry (naked eyes) (Feng et al., 2017; Gao et al., 2014). This review mainly discusses about the colorimetric methods using plasmonic nanostructures, so the non-colorimetric methods is not included.

In this review, we exclusively review recent advances in the development of "non-aggregation" colorimetric plasmonic sensors. To start with, we provide a brief introduction to the physical principles of LSPR. Then, we discuss "non-aggregation" plasmonic sensors from the aspects of strategies and applications, including plasmonic sensors based on metal nanoparticle etching, metal nanoparticle growth, as well as plasmonic colorimetric test strips. Finally, challenges and future directions in this fascinating research field are discussed.

\section{Physical principles of LSPR}

Localized surface plasmon resonance (LSPR) is an optical phenomenon generated by a light wave trapped within conductive nanoparticles, such as gold or silver nanoparticles (Ghosh and Pal, 2007; LizMarzán, 2004). The phenomenon is the result of interaction between the incident light and free electrons in the metal nanoparticles (Fig. 1A). This interaction produces coherent localized plasmon oscillations with a resonant frequency. The intensity and frequency of the plasmonic band are highly dependent on the size, shape, composition, dielectric environment, and distance of NPs from one another (Lee and El-Sayed, 2005; Nehl and Hafner, 2008). According to Mie theory, the extinction cross section, $\sigma_{\text {ext }}$, of a spherical particle with radius $\mathrm{R}$ (only when $R$ is much smaller than the wavelength $\lambda$ ) can be represented by (Liz-Marzán, 2004)

$\sigma_{\mathrm{ext}}=\frac{24 \pi^{2} R^{3}}{\lambda} \epsilon_{\mathrm{m}}^{3 / 2} \frac{\epsilon_{2}(\omega)}{\left[\epsilon_{1}(\omega)+2 \epsilon_{\mathrm{m}}\right]^{2}+\left[\epsilon_{2}(\omega)\right]^{2}}$

where $\omega$ is the frequency of incident light, $\lambda$ is the wavelength of light, $\epsilon$ $\mathrm{m}$ is the dielectric function of the surrounding medium, and $\epsilon(\omega)$ $=\epsilon_{1}(\omega)+\mathrm{i} \epsilon_{2}(\omega)$ is a dielectric function of the material itself. While $\epsilon_{\mathrm{m}}$ is assumed to be frequency independent, $\epsilon(\omega)$ is complex and a function of energy. The resonance condition is fulfilled when $\epsilon_{1}(\omega)=-2 \epsilon_{\mathrm{m}}(\omega)$ and $\epsilon_{2}(\omega)$ is weakly dependent on $\omega$.
The distance-dependent LSPR property is one of the most interesting properties of plasmonics, and this property can be applied to a lot of applications, such as biosensors (Elghanian et al., 1997) and plasmonenhanced spectroscopy (Talley et al., 2005). When the distance between nanoparticles is smaller than the size of the metal nanoparticles, the plasmon band shifts to a longer wavelength due to the substantial plasmon coupling effect. (Ghosh and Pal, 2007) As shown in Fig. 1B, the red-shift of plasmon band becomes more and more large with decreasing the distance of gold nanoparticle dimer (Zhong et al., 2004). By controlling the nanoparticles' aggregation, a lot of plasmonic colorimetric sensors have been fabricated (Elghanian et al., 1997; Saha et al., 2012; Zhou et al., 2015a). However, many parameters including salt, $\mathrm{pH}$, temperature, ligands, and solvent will affect the aggregation of nanoparticles, leading to the shift of plasmon band (Ghosh and Pal, 2007). Therefore, these interferences will limit the real applicability of "aggregation" plasmonic colorimetric sensors in complex samples.

Besides the distance-dependent property, LSPR is also sensitive to the size, shape, and dielectric environment of single metal nanoparticles (Kelly et al., 2003a; Nehl and Hafner, 2008). For instance, for gold spherical nanoparticles, a decrease in size leads to a decrease of plasmon absorption band (Fig. 1C), which can be attributed to the reudction of the extinction coefficient (see Eq. (1)) (Jana et al., 2001). The shape of nanoparticles has a significant effect on the plasmon band position because of the change of plasmon modes (Kelly et al., 2003a). As shown in Fig. 1D, the maximum plasmon band of silver nanoprisms alters to short wavelength when the sharp angles of silver nanoprisms become round and smooth (Yang et al., 2014). Gold nanorods have been widely used as plasmonic material due to their impressive optical property (Chen et al., 2013a). Gold nanorods have two plasmonic bands: a transverse mode band and a longitudinal mode band (Chen et al., 2013a). The longitudinal band is extremely sensitive to the aspect ratio (length/width) and will shift to a longer wavelength with increasing aspect ratio, resulting in a visible color change (Fig. 1E) (Kim et al., 2002). There is a derived relationship between the absorption maximum $\left(\lambda_{\max }\right)$ of the longitudinal plasmon resonance as a function of aspect ratio (R) and dielectric constant $\epsilon_{\mathrm{m}}\left(\epsilon_{\mathrm{m}}\right.$ is 1.77 in water): $\lambda_{\max }$ $=(53.71 . \mathrm{R}-42.29) \epsilon_{\mathrm{m}}+495.14$ (Link and El-Sayed, 2005). Also, the plasmonic band of core/shell bimetallic nanoparticles (e.g., Au@Ag) is sensitive to shell thickness due to a change in dielectric environment (Fig. 1F) (Okuno et al., 2010).

\section{Strategies for "non-aggregation" plasmonic colorimetric sensors}

"Non-aggregation" colorimetric plasmonic sensors can be divided into two types according to their sensing mechanism: Those that rely on the etching of metal nanoparticles and those that are based on the growth of metal nanoparticles (Scheme 1). Etching induces a change in

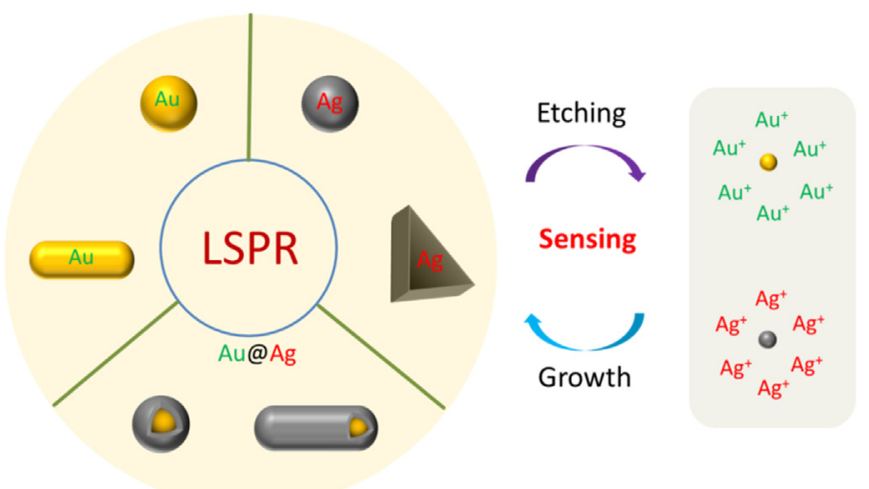

Scheme 1. Schematic of the sensing mechanisms of "non-aggregation" plasmonic sensors. 


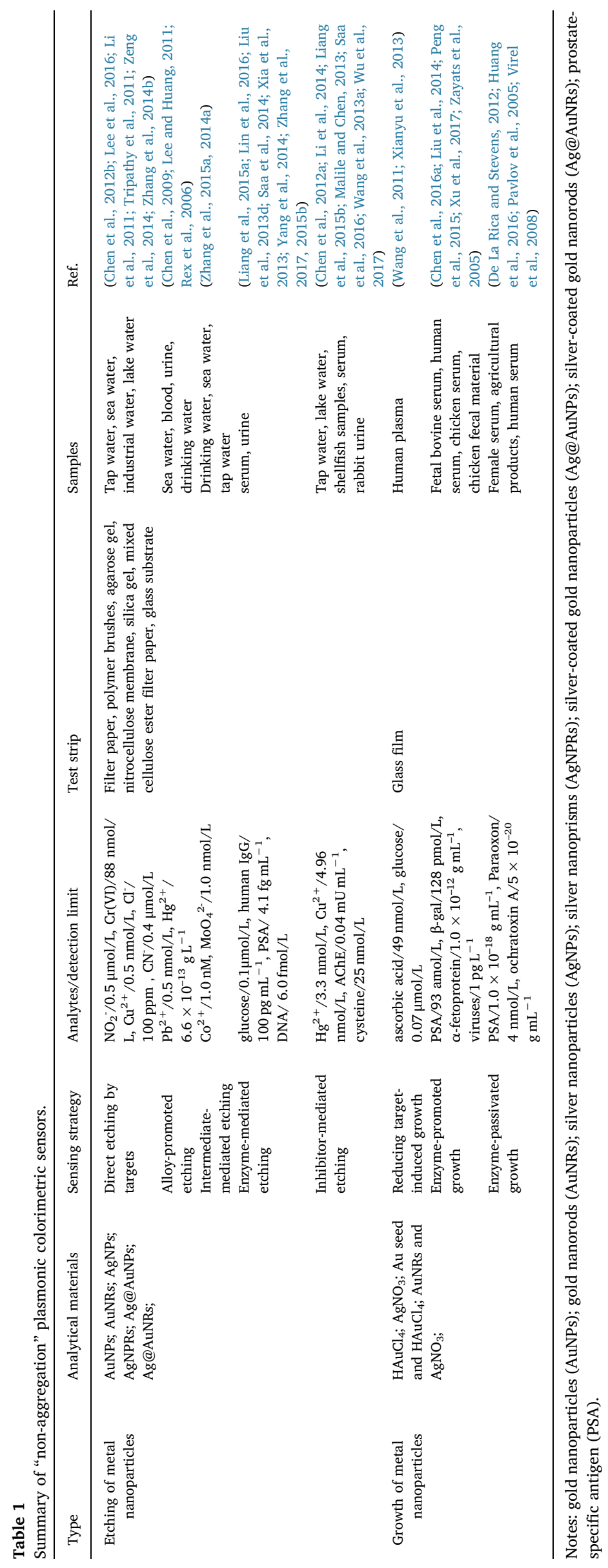




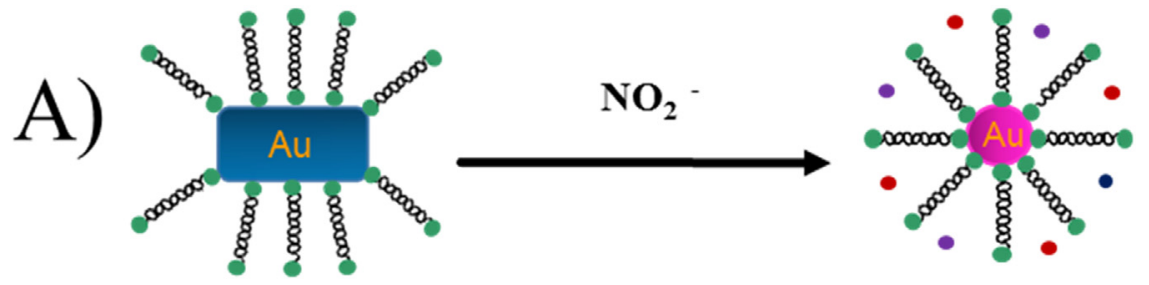

Fig. 2. Colorimetric plasmonic sensing based on direct etching of metal nanoparticles by targets. (A) Scheme for colorimetric detection of nitrite based on etching of gold nanorods. Reprinted (adapted) with permission from Chen et al. (2012b). Copyright: The Royal Society of Chemistry. (B) Schematic diagram of Au@Ag core/shell NPs for colorimetric detection of cyanide. Reprinted (adapted) with permission from Zeng et al. (2014). Copyright: The Royal Society of Chemistry.

$00000000: \mathrm{CTAB} \quad 0: \mathrm{AuCl}_{2}^{-}-\left(\mathrm{CTA}^{+}\right)_{2} \quad 0: \mathrm{AuBr}_{2}^{-}-\left(\mathrm{CTA}^{+}\right)_{2}$

B)

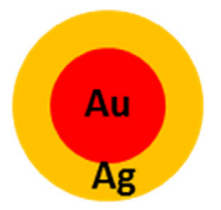

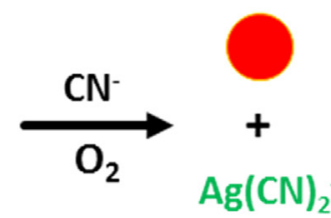

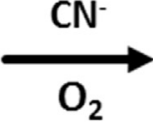

$\mathrm{Au}(\mathrm{CN})_{2}^{-}$

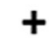

$\mathrm{Ag}(\mathrm{CN})_{2}$ nanoparticle size, shape, or composition; as a result, there is a change in the LSPR extinction spectroscopy of the nanoparticles and a subsequent change in color (Kelly et al., 2003b). The growth of nanoparticles refers to the generation of plasmonic nanoparticles or new plasmonic nanostructures from metal ions in the absence/presence of seeds, leading to the appearance or changes in the LSPR band extinction spectra, along with solution color changes. Highly sensitive and selective detection of various targets, including metal ions, anions, biomarkers, bacteria, and viruses, has been achieved using "non-aggregation" plasmonic sensors in recent years. Some typical examples are shown in Table 1. Because of their high stability, many of the sensors are used for detection of targets in complex matrices, such as serum and seawater. Also, some have been developed into test strips (Ferhan et al., 2013; Zhang et al., 2014b), which are more practical for real-world applications. In this section, we focus on sensing strategies of "non-aggregation" plasmonic colorimetric sensors as well as test strips.

\subsection{Plasmonic colorimetric sensors using metal nanoparticle etching}

To date, different metal nanoparticles, such as gold nanospheres, gold nanorods, silver nanospheres, silver nanoprisms, core/shell Au@Ag nanospheres, and core/shell Au@Ag nanorods, have been used as etching-based plasmonic sensors. Etching-based plasmonic sensors incorporating these nanoparticles have been used to detect various targets including DNA, (Ma et al., 2016; Malile and Chen, 2013; Yang et al., 2014) proteins, (Cheng et al., 2016; Guo et al., 2016b; Liang et al., 2015a; Lu et al., 2016; Yang et al., 2017; Zhang et al., 2015b; Zhao et al., 2016) metal ions, (Chang et al., 2017; Chemnasiri and Hernandez, 2012; Chen et al., 2016b, 2016c, 2009, 2013b; Jin and Han, 2014; Lan and Lin, 2014; Liu et al., 2013c; Wang et al., 2014; Yao et al., 2017; Zhang et al., 2014a, 2014b; Zhu et al., 2016) anions, (Chen et al., 2015, 2012b; He and Yu, 2015; Li et al., 2011, 2015; Liu et al., 2013a; Sasikumar and Ilanchelian, 2017; Tripathy et al., 2011; Xin et al., 2012; Zeng et al., 2014; Zhang et al., 2015a, 2016) and glucose, (Jang and Min, 2015; Lin et al., 2016; Liu et al., 2013d; Saa et al., 2014; Xia et al., 2013; Zhang et al., 2017), amino acids, (Li et al., 2014) and small organic molecules (Fang et al., 2017; Gu et al., 2016; Liu et al., 2013b; Liu et al., 2018; Oh et al., 2014; Saa et al., 2016; Tan et al., 2014; Wang et al., 2013b; Wu et al., 2015a; Yang et al., 2015). By sensing strategy, five main types of plasmonic sensors can be recognized: direct etching by targets, alloy-promoted etching, intermediate-mediated etching, enzyme-mediated etching, and inhibitor-mediated etching.

\subsubsection{Direct etching by targets}

Usually, Au and Ag are quite stable and are difficult to oxidize (etch) because of their high redox potential. However, the redox potential of
$\mathrm{Au}$ and $\mathrm{Ag}$ can be decreased under some conditions, such as in the presence of appropriate ligands (e.g., $\mathrm{CN}^{-}, \mathrm{Cl}^{-}$, and $\mathrm{SCN}^{-}$). Also, redox potential also decreases when a bulk material is made into nanoparticles because of the increase in active surface. Based on these properties, targets that have a relatively high redox potential or can act as complexing agents of novel metal ions (e.g., $\mathrm{Au}^{+}$and $\mathrm{Ag}^{+}$) can be detected by inducing the etching of $\mathrm{Au}$ or $\mathrm{Ag}$ nanoparticles. This strategy has been used for determination of $\mathrm{NO}_{2}^{-}, \mathrm{Cr}(\mathrm{VI}), \mathrm{Cu}^{2+}, \mathrm{Cl}^{-}$, and $\mathrm{CN}^{-}$ions in complex samples such as seawater and urine. Also, some plasmonic sensors (e.g., $\mathrm{Cu}^{2+}$ (Zhang et al., 2014b) and $\mathrm{Pb}^{2+}$ (Ferhan et al., 2013)) have been developed into colorimetric test strips.

As a typical example, the Chen group developed a label-free colorimetric method for nitrite detection based on the etching of gold nanorods under acidic conditions (Fig. 1A). (Chen et al., 2012b) The example demonstrates a target with oxidizing ability. Under acidic conditions, the presence of $\mathrm{Cl}^{-}, \mathrm{Br}^{-}$, and cetyltrimethylammonium $\left(\mathrm{CTA}^{+}\right)$can decrease the redox potential of $\mathrm{Au}(\mathrm{I}) / \mathrm{Au}$ dramatically due to the coordination effect. In contrast, $\mathrm{NO}_{2}^{-}$has a relatively high redox potential under acidic conditions. Therefore, gold nanorods were oxidized into $\mathrm{AuCl}_{2}^{-} \cdot\left(\mathrm{CTA}^{+}\right)_{2}$ and $\mathrm{AuBr}_{2}^{-} \cdot\left(\mathrm{CTA}^{+}\right)_{2}$ quickly. As a result, the aspect ratio of the gold nanorods decreased, accompanied by a plasmonic band shift as well as a distinct change in color. The band shift and color change showed a good correlation with the concentration of $\mathrm{NO}_{2}$. The optimal detection time of this method is $20 \mathrm{~min}$. The visual detection limit was $4 \mu \mathrm{mol} / \mathrm{L}$, which is far below the maximum containment level recommended by the U.S. Environmental Protection Agency (EPA; about $71 \mu \mathrm{mol} / \mathrm{L}$ ). This method was used to detect $\mathrm{NO}_{2}{ }^{-}$ in drinking water. Sensitive and selective detection of $\mathrm{Cu}^{2+}$ and $\mathrm{Cr}(\mathrm{VI})$ using this strategy has also been achieved (Li et al., 2011; Zhang et al., 2014b).

As mentioned above, targets that can decrease the redox potential of nanoparticles can be detected using this strategy. For example, Zeng and coworkers developed a simple, sensitive, and selective plasmonic sensor for $\mathrm{CN}^{-}$based on etching of $\mathrm{Au} @ \mathrm{Ag}$ core/shell nanostructures (Fig. 2B) (Zeng et al., 2014). Here, $\mathrm{CN}^{-}$acted as the ligand of $\mathrm{Ag}^{+}$and $\mathrm{Au}^{+}$and decreased the redox potential of $\mathrm{Ag}^{+} / \mathrm{Ag}$ and $\mathrm{Au}^{+} / \mathrm{Au}$. In the presence of $\mathrm{CN}^{-}, \mathrm{Au} @ \mathrm{Ag}$ core/shell nanoparticles were gradually etched by dissolved oxygen. Etching of the silver shell occurred first, followed by etching of the Au core. As a result, the color of the solution changed from yellow to red to colorless. This method showed excellent sensitivity, with a detection limit of $0.5 \mu \mathrm{mol} / \mathrm{L}$, fast response (less than one minute), as well as excellent selectivity. Similarly, Kim reported a colorimetric method for the detection of $\mathrm{Cl}^{-}$ions in aqueous environments based on the ability of $\mathrm{Cl}^{-}$to complex with $\mathrm{Au}^{+}$. The presence of $\mathrm{Cl}^{-}$ caused rapid etching of gold nanoparticles in the presence of strong oxidizing agents, such as $\mathrm{HNO}_{3}$ or $\mathrm{H}_{2} \mathrm{O}_{2}$ (Tripathy et al., 2011). The 

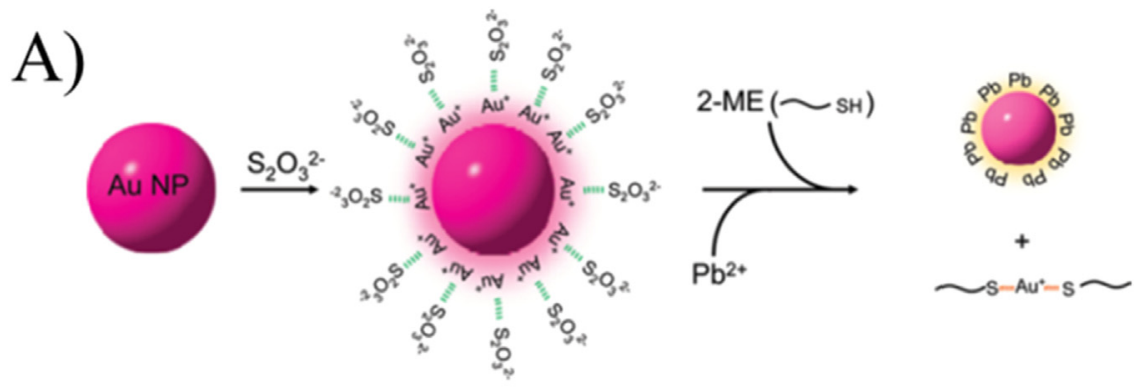

Fig. 3. Colorimetric plasmonic sensing based on alloypromoted etching of metal nanoparticles by targets. (A) Scheme for colorimetric detection of $\mathrm{Pb}^{2+}$ based on etching of gold nanoparticles. Reprinted (adapted) with permission from Chen et al. (2009). Copyright: The American Chemical Society. (B) Schematic showing the amalgamation of $\mathrm{Hg}$ with $\mathrm{Au}$ nanorods. Reprinted (adapted) with permission from Rex et al. (2006). Copyright: The American Chemical Society.
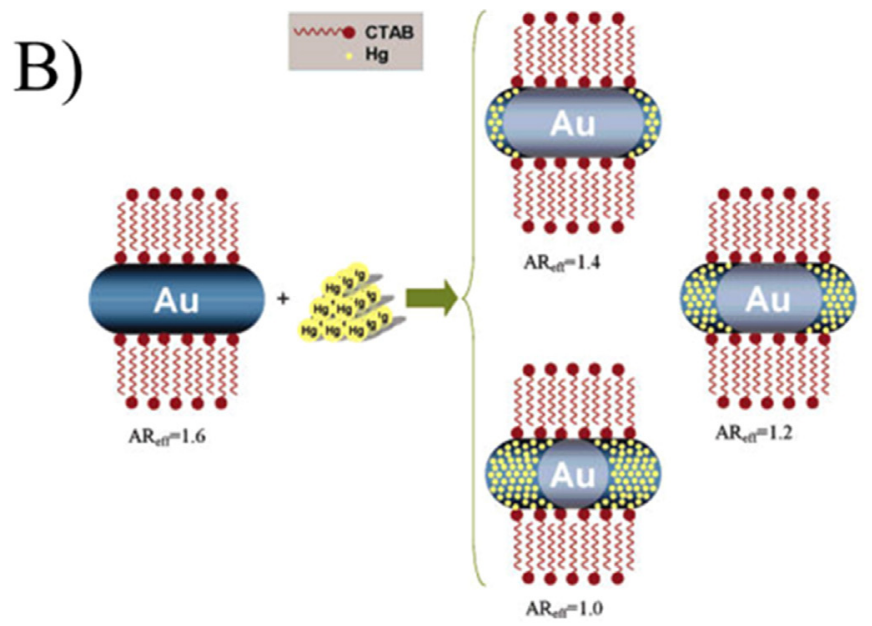

etching process led to remarkable damping of the LSPR peak of the gold nanoparticles at around $520 \mathrm{~nm}$, along with a color change from red to colorless. The detection limit was $500 \mathrm{ppm}$ (around $14.2 \mathrm{mmol} / \mathrm{L}$ ) in natural water systems. This method was highly selective for $\mathrm{Cl}^{-}$in the presence of several common mineral acids, salts, and anions. Since the detection of intracellular $\mathrm{Cl}^{-}$anion is important and it is difficult to measure it directly (Siegel et al., 1976), therefore, this method may open up a new field of applications.

\subsubsection{Alloy-promoted etching}

Another way to develop etching-based plasmonic sensors is to accelerate the etching of gold nanoparticles by the formation of alloys on the nanoparticles (Chen et al., 2009; Lee and Huang, 2011). Chang's group reported a colorimetric and label-free plasmonic probe for the detection of $\mathrm{Pb}^{2+}$ in aqueous solution based on the accelerated etching of gold nanoparticles by $\mathrm{Pb}^{2+}$ ions in the presence of thiosulfate $\left(\mathrm{S}_{2} \mathrm{O}_{3}{ }^{2-}\right)$ and 2-mercaptoethanol (Fig. 3A) (Chen et al., 2009). The formation of $\mathrm{Pb}$-Au alloys on the surfaces of the gold nanoparticles in the presence of $\mathrm{Pb}^{2+}$ ions and 2-ME was validated by surface-assisted laser desorption/ionization time-of-flight ionization mass spectrometry. After the formation of $\mathrm{Pb}$-Au alloys, the gold nanoparticles dissolved rapidly into solution, which resulted in a dramatic decrease in LSPR absorption, accompanied by a color change. This approach was highly sensitive, with a detection limit of $0.5 \mathrm{nmol} / \mathrm{L}$, and was selective for $\mathrm{Pb}^{2+}$ ions. Practical application of this method was illustrated by successful determination of the concentrations of $\mathrm{Pb}^{2+}$ ions in real environmental samples. The method allows for the detection at room temperature, but needs about $1.8 \mathrm{~h}$ for the test. The Campiglia group developed a colorimetric method for $\mathrm{Hg}^{2+}$ detection based on amalgamation induced-shape transformation of gold nanorods (Fig. 3B) (Rex et al., 2006). An amalgam formed on gold nanorods in the presence of sodium borohydride and $\mathrm{Hg}^{2+}$. The formation of amalgam resulted in shape transformation of the gold nanorods from rods to spheres, causing a blue-shift of the LSPR as well a color change. The reported
B

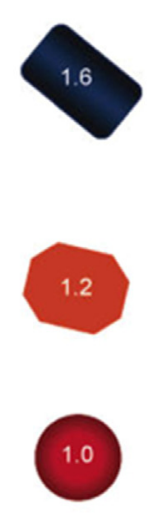

limit of detection $\left(6.6 \times 10^{-13} \mathrm{~g} / \mathrm{L}\right)$ indicates that this sensor can potentially be used to monitor ultralow levels of mercury in water samples. The detection procedure was simple and each sample only required less than $10 \mathrm{~min}$. The reaction solution was mixed in a test tube, which is well suited for on-site detection.

\subsubsection{Intermediate-mediated etching}

In this strategy, the target can selectively trigger the generation of reactive intermediates that then act as etching agent (i.e., oxidizing or complexing agents). This concept was first proposed by the Chen group (Zhang et al., 2015a, 2014a), who established a visual detection method for $\mathrm{Co}^{2+}$ ions based on the Fenton-like reaction-mediated etching of gold nanorods (Fig. 4A) (Zhang et al., 2014a). In the presence of bicarbonate $\left(\mathrm{HCO}_{3}{ }^{-}\right)$and hydrogen peroxide $\left(\mathrm{H}_{2} \mathrm{O}_{2}\right), \mathrm{Co}^{2+}$ ions triggered a Fenton-like reaction, which generated large amounts of active oxidant superoxide radicals $\left(\mathrm{O}_{2}{ }^{-}\right)$. Simultaneously, the gold nanorods were etched by $\mathrm{O}_{2}^{-{ }^{-}}$in the presence of $\mathrm{SCN}^{-}$, resulting in an apparent color change from green to red. Under optimized conditions, excellent analytical performance for $\mathrm{Co}^{2+}$ was obtained with a lower detection limit of $1.0 \mathrm{nmol} / \mathrm{L}$. Importantly, the very low visual detection limit of $40 \mathrm{nmol} / \mathrm{L}$ makes possible on-site rapid detection by the naked eye. This method has a quick response with $7 \mathrm{~min}$, however, requiring high temperature at around $90^{\circ} \mathrm{C}$.

As another example, the Chen group also developed a novel approach for visual detection of molybdate $\left(\mathrm{MoO}_{4}{ }^{2-}\right)$ in aqueous media (Fig. 4B) (Zhang et al., 2015a). Their method is based on the combination of a catalytic formation of iodine and iodine-mediated etching of gold nanorods. Molybdate catalyzes the reaction between $\mathrm{H}_{2} \mathrm{O}_{2}$ and $\mathrm{I}^{-}$ to generate $\mathrm{I}_{2}$. Then, the gold nanorods are etched by $\mathrm{I}_{2}$ preferentially in the longitudinal direction in the presence of CTAB. The etching results in a blue shift of the longitudinal LSPR of the gold nanorods, accompanied by a color change from blue to red. The incubation time for the detection is $15 \mathrm{~min}$ at $50^{\circ} \mathrm{C}$. This sensor showed excellent sensitivity, with a detection limit of $1.0 \mathrm{nmol} / \mathrm{L}$. Also, its high selectivity and 

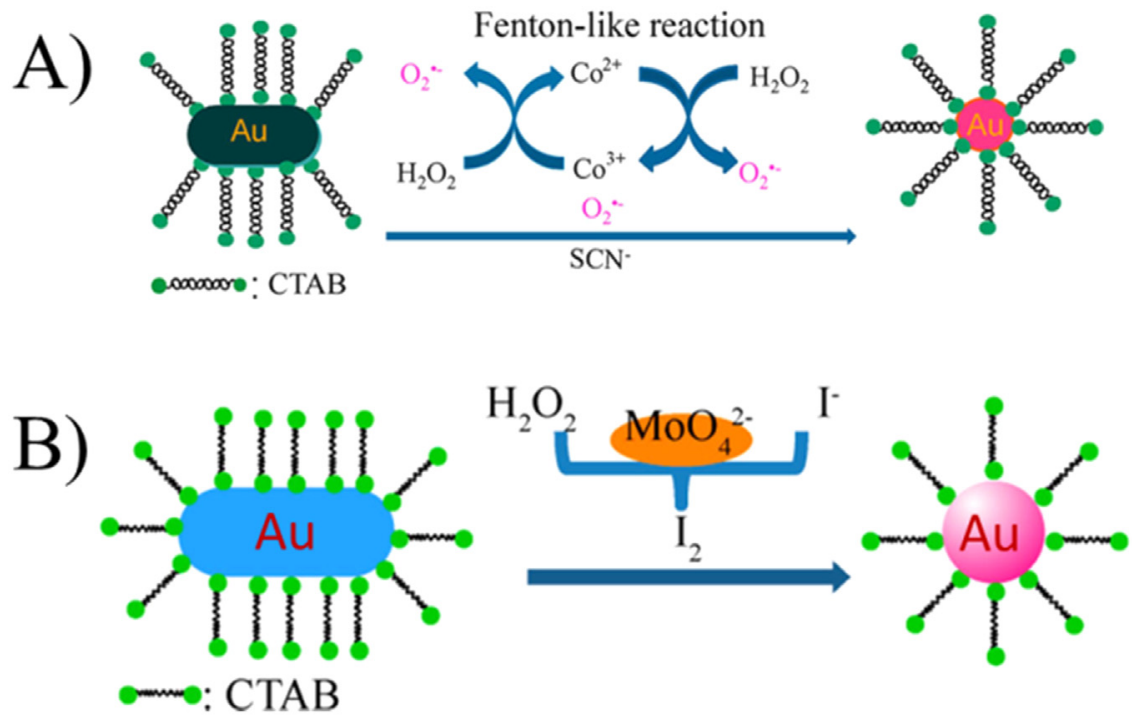

Fig. 4. Colorimetric plasmonic sensing based on intermediate-mediated etching of metal nanoparticles. (A) Schematic illustration of visual detection of $\mathrm{Co}^{2+}$ based on Fenton-like reaction-mediated etching of gold nanorods. Reprinted with permission from Zhang et al. (2014a). Copyright: The American Chemical Society. (B) Schematic illustration of visual detection of molybdate based on enzymatic-like etching of gold nanorods. Reprinted with permission from Zhang et al. (2015a). Copyright: The American Chemical Society. tolerance to interference enabled the sensor to detect molybdate directly in real-world complex samples, such as tap water, drinking water, and seawater.

\subsubsection{Enzyme-mediated etching}

Enzyme-mediated etching-based plasmonic sensing is a subgroup of "intermediate-mediated etching." Considering the extensive use of enzymes, however, we treat this as an independent type of plasmonic sensor. This strategy involves the use of an enzyme to catalyze a reaction to produce products that can induce etching of metal nanoparticles. Because the enzyme can be modified to label DNA and antibodies, this strategy can be further applied to plasmonic DNA assays and enzymelinked immunosorbent assays (ELISAs). This approach can therefore significantly expand the range of targets depending on the availability of corresponding DNA probes or antibodies.

Glucose monitoring is of great interest because of the increasing incidence of diabetes worldwide, and plasmonic sensors to detect glucose have been designed. For example, the Xia group invented a colorimetric method for simple, sensitive, and low-cost colorimetric sensing of glucose in serum based on glucose oxidase (GOx)-mediated etching of Ag nanoprisms (Fig. 5A) (Xia et al., 2013). The detection procedure was carried out at room temperature for $15 \mathrm{~min}$. In the presence of $\mathrm{GOx}, \mathrm{H}_{2} \mathrm{O}_{2}$ was produced by the enzymatic oxidation of glucose. Silver triangular-shaped nanoprisms were then etched by the generated $\mathrm{H}_{2} \mathrm{O}$, resulting in a shape change to round. With increasing glucose, the longitudinal LSPR peak shifted to a shorter wavelength with a color change from blue to red. The shift of the LSPR and the resulting color change can be used for semi-quantitative and quantitative detection of glucose by the naked eye. The detection limit was 0.2 $\mu \mathrm{mol} / \mathrm{L}$. Due to its ultrahigh sensitivity, only $10-20 \mu \mathrm{L}$ of serum was sufficient for a one-time determination. Chen's group developed a highly sensitive colorimetric method for glucose in human urine with a detection limit of $0.1 \mu \mathrm{mol} / \mathrm{L}$ based on enzyme-like-reaction-mediated etching of gold nanorods (Zhang et al., 2017). The advantage of this method is to avoid the complex pretreatment of urine sample.

DNA assays and ELISAs are widely used biological assays. The development of plasmonic DNA assays and ELISAs will significantly enlarge the application fields of etching-based plasmonic sensing. The Gao group developed a simple DNA detection platform based on the etching of silver nanoprisms (Fig. 5B) (Yang et al., 2014). Here, GOx was used as the labeling enzyme for amplification. The $\mathrm{H}_{2} \mathrm{O}_{2}$ generated from the GOx-catalyzed reaction-etched silver nanoprisms and led to a shift in the LSPR band. Through enzyme-linked hybridization chain reaction amplification, this assay was able to detect $6.0 \mathrm{fmol} / \mathrm{L}$ target DNA. The
Chen group established a plasmonic ELISA based on colorimetric detection of alkaline phosphatase (ALP), with the color change triggered by iodine-mediated etching of gold nanorods (Fig. 5C) (Zhang et al., 2015b). As the product of the ALP-triggered reaction, iodine etched rod-shaped gold nanorods into spheres, resulting in a color change from blue to red due to blue-shift of the longitudinal LSPR of gold nanorods. Human immunoglobulin G (IgG) was chosen as the protein target in the study of Chen and colleagues. The color change and peak shift were used for quantification of human IgG. Under optimal conditions, the detection limit for IgG was $100 \mathrm{pg} / \mathrm{mL}$, which is a much lower detection limit than that of conventional ELISA. Also, successful detection of human IgG in fetal bovine serum indicated that this method was applicable to the determination of low abundance proteins in complex biological samples.

\subsubsection{Inhibitor-mediated etching}

Some inhibitors can hinder etching of metal nanoparticles. Under these conditions, etching rate can be altered by controlling the concentration of the inhibitor. Two specific strategies have been applied: inhibition of etching by targets and reactivation of inhibited etching (Fig. 6). The Chen group developed a gold nanorod-based method to detect $\mathrm{Cu}^{2+}$ ions based on catalytic decomposition of $\mathrm{H}_{2} \mathrm{O}_{2}$ (Fig. 6A) (Wang et al., 2013a). $\mathrm{H}_{2} \mathrm{O}_{2}$ was employed to etch gold nanorods, leading to a blue-shift of the longitudinal LSPR along with a color change of the solution from bluish green to purplish red. The presence of $\mathrm{Cu}^{2+}$ catalyzed the decomposition of $\mathrm{H}_{2} \mathrm{O}_{2}$ and significantly inhibited the etching rate. This assay demonstrated an excellent linear range from 10 to $300 \mathrm{nmol} / \mathrm{L}$, and the visual detection limit was 10 $\mathrm{nmol} / \mathrm{L}$. This approach is operated at $60^{\circ} \mathrm{C}$ and $17 \mathrm{~min}$ is needed for incubation of one sample. This method has been successfully used to detect $\mathrm{Cu}^{2+}$ in shellfish samples, indicating its practical applicability. The Chen group also reported a colorimetric method to detect $\mathrm{Hg}^{2+}$ ions based on reactivation of inhibited etching of gold nanorods by abstraction of the protection ligand on silver nanoprisms (Fig. 6B) (Chen et al., 2012a). Here, 1-dodecanethiol $\left(\mathrm{C}_{12} \mathrm{H}_{25} \mathrm{SH}\right.$ )-capped silver nanoprisms were used to inhibit the etching of silver nanoprisms by $\mathrm{I}^{-}$. In the presence of $\mathrm{Hg}^{2+}, \mathrm{C}_{12} \mathrm{H}_{25} \mathrm{SH}$ was abstracted by $\mathrm{Hg}^{2+}$ from the surface of the silver nanoprisms; as a result, silver nanoprisms without ligands were etched by $\mathrm{I}^{-}$, which was accompanied by a rapid color change from green to red. With the increase in $\mathrm{Hg}^{2+}$, the LSPR band of silver nanoprisms blue-shifted and exhibited an excellent linear relationship in the range of $10-500 \mathrm{nmol} / \mathrm{L}$. Also, excellent selectivity toward $\mathrm{Hg}^{2+}$ was observed. This method was used to quantify $\mathrm{Hg}^{2+}$ in tap water, drinking water, and lake water, with satisfactory recoveries 

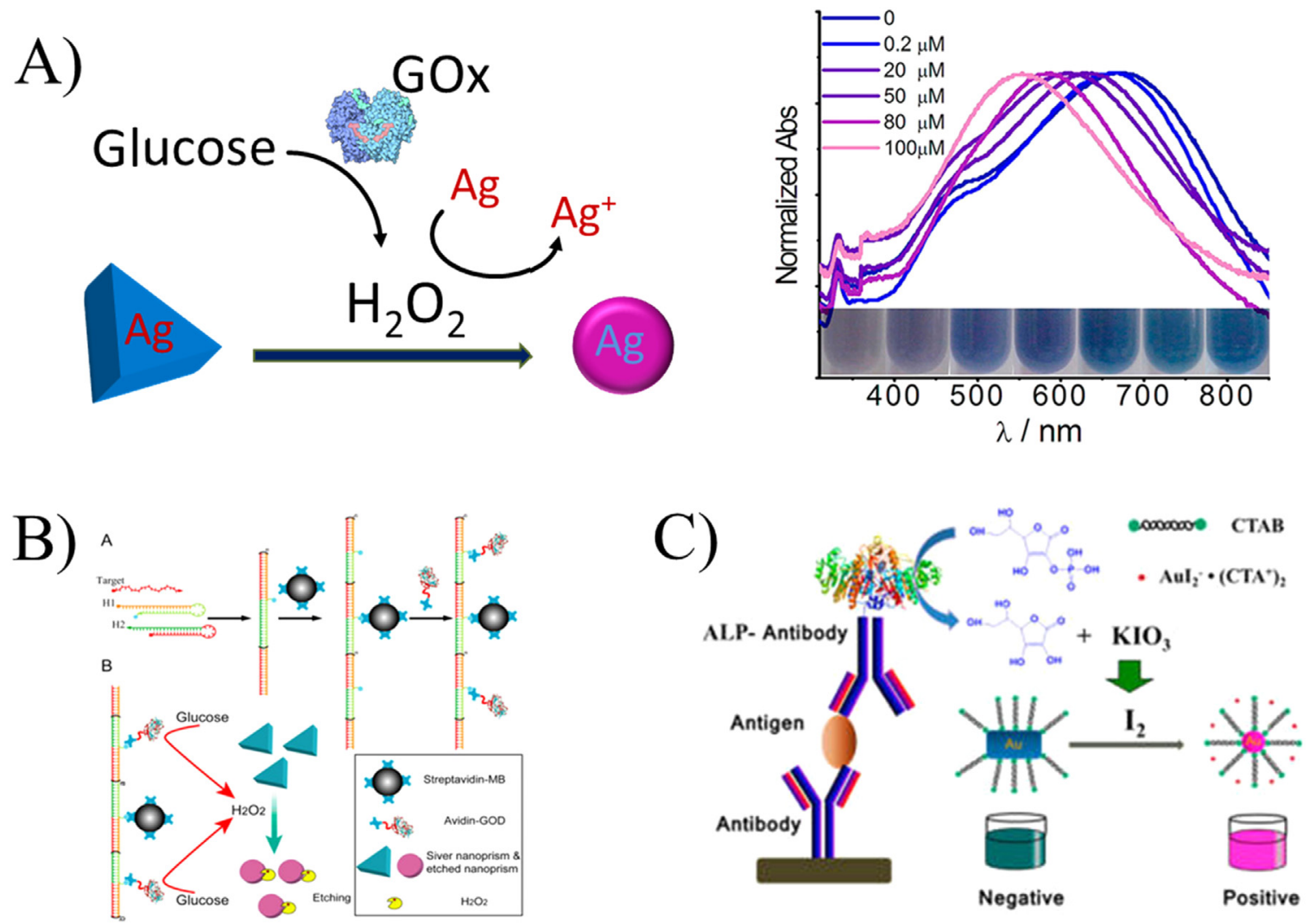

Fig. 5. Colorimetric plasmonic sensing based on enzyme-mediated etching of metal nanoparticles. (A) Schematic illustration of an Ag nanoprism-GOx homogenous system for colorimetric sensing of glucose. Reprinted with permission from Xia et al. (2013). Copyright: The American Chemical Society. (B) Construction of specific DNA detection platform based on etching of triangular silver nanoprisms. Reprinted with permission from Yang et al. (2014). Copyright: The American Chemical Society. (C) Schematic representation of a visual plasmonic ELISA based on iodine-mediated etching of gold nanorods. Reprinted with permission from Zhang et al. (2015b). Copyright: The American Chemical Society.

reported for these sample types. This method has a quick response time $(15 \mathrm{~min})$ and all the procedure occur at room temperature, which makes the on-site detection possible.

\subsection{Plasmonic colorimetric sensors based on metal nanoparticle growth}

$\mathrm{AuCl}_{4}{ }^{-}$and $\mathrm{Ag}^{+}$are normally used as precursors for plasmonic nanoparticles in growth-based plasmonic sensing. Seeds are also sometimes used for the growth of nanoparticles. Growth-based plasmonic sensors have been used to detect different analytes including DNA, (Guo et al., 2016a) glucose, (Xianyu et al., 2013; Xiong et al., 2015) enzymes, (Guo et al., 2016c; Tang et al., 2015; Wang et al., 2015; Yan et al., 2015) biomarkers, (Cecchin et al., 2014; De La Rica and Stevens, 2012, 2013; Li et al., 2016; Ma et al., 2017; Peng et al., 2015;
A)

$\mathrm{H}_{2} \mathrm{O}_{2} \quad$ No Reaction
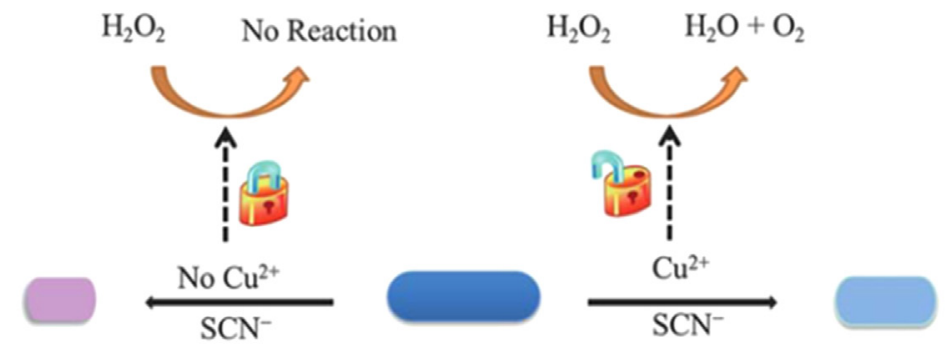

B)
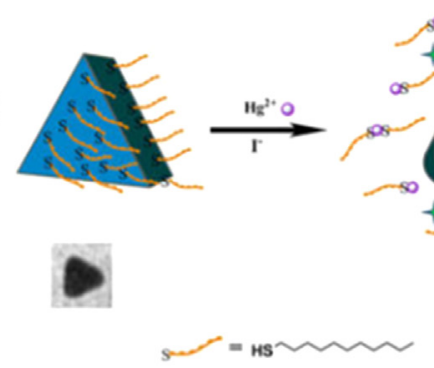

$\mathrm{O}=\mathrm{Hg}^{2+}$
Fig. 6. Colorimetric plasmonic sensing based on inhibitormediated etching of metal nanoparticles. (A) Schematic mechanism for $\mathrm{Cu}^{2+}$ sensing based on decelerated etching of gold nanorods. Reprinted with permission from Wang et al. (2013a). Copyright: The Royal Society of Chemistry. (B) Schematic sensing mechanism of a $\mathrm{Hg}^{2+}$-controlled morphology transition of 1-dodecanethiol-capped Ag nanoprisms in the presence of excess $\mathrm{I}^{-}$. Reprinted with permission from Chen et al. (2012a). Copyright: The American Chemical Society. 

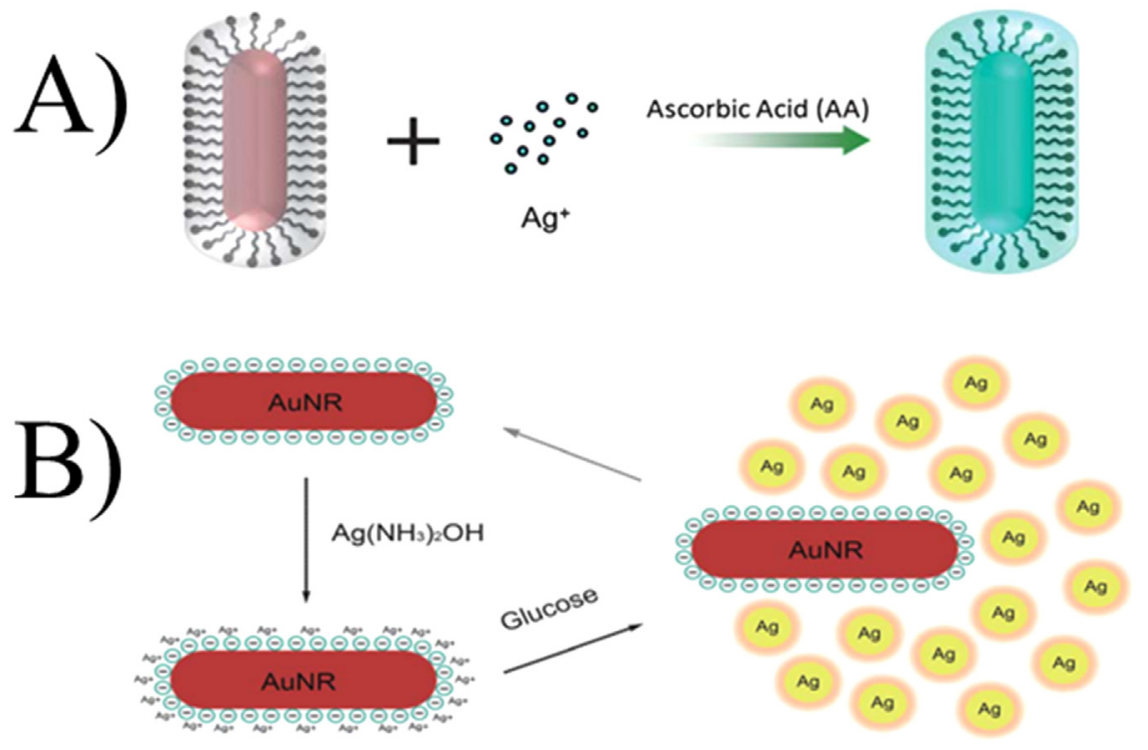

Fig. 7. Colorimetric plasmonic sensing based on direct growth of metal nanoparticles. (A) Schematic representation of the LSPR sensing mechanism of AA using MSGNR probes in the presence of $\mathrm{Ag}^{+}$. Reprinted with permission from Wang et al. (2011). Copyright: The Royal Society of Chemistry. (B) Schematic illustration of a colorimetric, non-enzymatic glucose assay based on the gold nanorodassisted production of silver nanoparticles (GAPS). Reprinted with permission from Xianyu et al. (2013). Copyright: The Royal Society of Chemistry.
Xuan et al., 2016; Yang and Gao, 2015) viruses, (Xu et al., 2017; Zhou et al., 2014) bacteria, (Chen et al., 2016a; Yu et al., 2017) nerve gas, (Pavlov et al., 2005) and small organic molecules (Huang et al., 2016; Soh et al., 2015; Wang et al., 2011; Zhou et al., 2016). Formation or metallization of plasmonic nanoparticles by reducing agents results in a color change. By this principle, current growth-based plasmonic sensors can be divided into three categories: reduction of target-induced growth, enzyme-induced growth, and inhibition of enzyme-induced growth.

\subsubsection{Reducing target-induced growth}

Some reducing targets can directly react with $\mathrm{AuCl}_{4}{ }^{-}$and $\mathrm{Ag}^{+}$to generate metal nanoparticles and this reaction can be used to sense the targets with reducing property. For example, the Chen group developed a growth-based plasmonic colorimetric assay of ascorbic acid by growing $\mathrm{Ag}$ on mesoporous silica-coated gold nanorods (Fig. 7A). (Wang et al., 2011) In the presence of ascorbic acid, silver was deposited on silica-coated gold nanorods, and the longitudinal LSPR band of silica-coated gold nanorods shifted gradually to a shorter wavelength with an increase in ascorbic acid. The detection process needs 20 min for the incubation of the sample. The detection limit of the developed method was $49 \mathrm{nmol} / \mathrm{L}$, which is comparable to that of fluorimetric methods. Similarly, the Jiang group reported a colorimetric, non-enzymatic glucose assay based on the growth of silver shells on gold nanorods (Fig. 7B). (Xianyu et al., 2013) The growth of gold nanorods caused blue-shift of the longitudinal LSPR band and a color change with increasing glucose concentration (Fig. 6B). This method is conducted at $80^{\circ} \mathrm{C}$ for around $8 \mathrm{~min}$. An excellent sensitivity, with a detection limit of $70 \mathrm{nmol} / \mathrm{L}$, was achieved, which is much lower than commercial kits.

\subsubsection{Enzyme-promoted growth}

Many enzymes, such as glucose oxidase (Liu et al., 2014; Zayats et al., 2005), alkaline phosphatase (Gao et al., 2014), tyrosinase (Baron et al., 2005), and NAD(P) ${ }^{+}$-dependent enzymes (Peng et al., 2015), can catalyze the decomposition of non-reducing substrates into reducing products. Thus, these enzymes can mediate the formation of metal nanoparticles by redox chemistry in the presence of metal ions. The formation of plasmonic nanoparticles induced by the generated reducing product can be used for many enzyme-related assays, such as glucose detection, enzyme activity assays and ELISAs. As an example, Willner's group developed a biosensor for glucose based on $\mathrm{H}_{2} \mathrm{O}_{2^{-}}$mediated enlargement of Au nanoparticles (Fig. 8) (Zayats et al., 2005). Here, due to the catalytic action of glucose oxidase (GOx), $\mathrm{H}_{2} \mathrm{O}_{2}$ generated in the presence of $\mathrm{O}_{2}$ /glucose enabled the growth of small gold nanoparticles, resulting in a color change. On modified glass slides, a limit of detection of $2 \mu \mathrm{mol} / \mathrm{L}$ was obtained. The method has fast response time and the detection for each sample only needs $10 \mathrm{~min}$.

The primary application of enzyme-promoted growth of metal nanoparticles is plasmonic ELISA. For example, Liu and coworker developed a plasmonic ELISA based on the GOx-catalyzed growth of $5 \mathrm{~nm}$ gold nanoparticles in the presence of $\mathrm{Au}^{3+}$, which allowed detection of cancer biomarkers at the attomolar level in clinical samples (Fig. 9A) (Liu et al., 2014). This method detected prostate-specific antigen at $93 \mathrm{aM}$, which is four orders of magnitude more sensitive than corresponding commercial ELISA. Also, the apparent color change induced by enzyme-induced growth of gold nanoparticles lends itself to point-ofcare diagnostics in both resource-rich and resource-limited areas.

Guo group utilized gold nanobipyramids (Au NBPs) with sharp edges in a growth-based plasmonic ELISA for ultrasensitive colorimetric detection of the H5N1 virus (Fig. 9B) (Xu et al., 2017). Alkaline phosphatase (ALP) catalyzed the decomposition of 4-aminophenyl phosphate to generate 4-aminophenol, a reductant, which then reduced silver ions into elemental silver on Au NBPs. The deposited silver caused a change in the refractive index of gold, along with a blue shift of the LSPR and a vivid color change. The proposed detection method for ALP was linear in the range of $0.1-5 \mathrm{mU} / \mathrm{mL}$, with a limit of detection of $0.086 \mathrm{mU} / \mathrm{mL}$. When the proposed method was used for plasmonic ELISA detection of the H5N1 virus, a detection limit of $1 \mathrm{pg} /$ $\mathrm{mL}$ was reported, indicating that the ELISA was much more sensitive than most reported colorimetric biosensors. Successful detection of the H5N1 virus in real samples with good recoveries suggests that this ELISA has practical utility.

\subsubsection{Enzyme-passivated growth}

Some enzymes can catalyze reactions that will either produce some product (inhibitor) that blocks the growth of nanoparticles or directly decomposes the reducing agent, thereby decreasing the growth rate of nanoparticles. For example, Pavlov et al. reported a simple and sensitive colorimetric assay for the detection of acetylcholine esterase (AChE) inhibitors by mediating the growth of Au-Ag core/shell nanoparticles (Fig. 10A) (Virel et al., 2008). AChE catalyzed the hydrolysis of acetylthiocholine to yield the thiol-bearing compound thiocholine. At low concentrations, thiocholine blocked the growth of silver shells on gold nanoparticles in the presence of ascorbic acid. In the presence of an inhibitor of the enzyme, yields of thiocholine were lower; as a result, the growth of Au-Ag nanoparticles was promoted. The shift in 

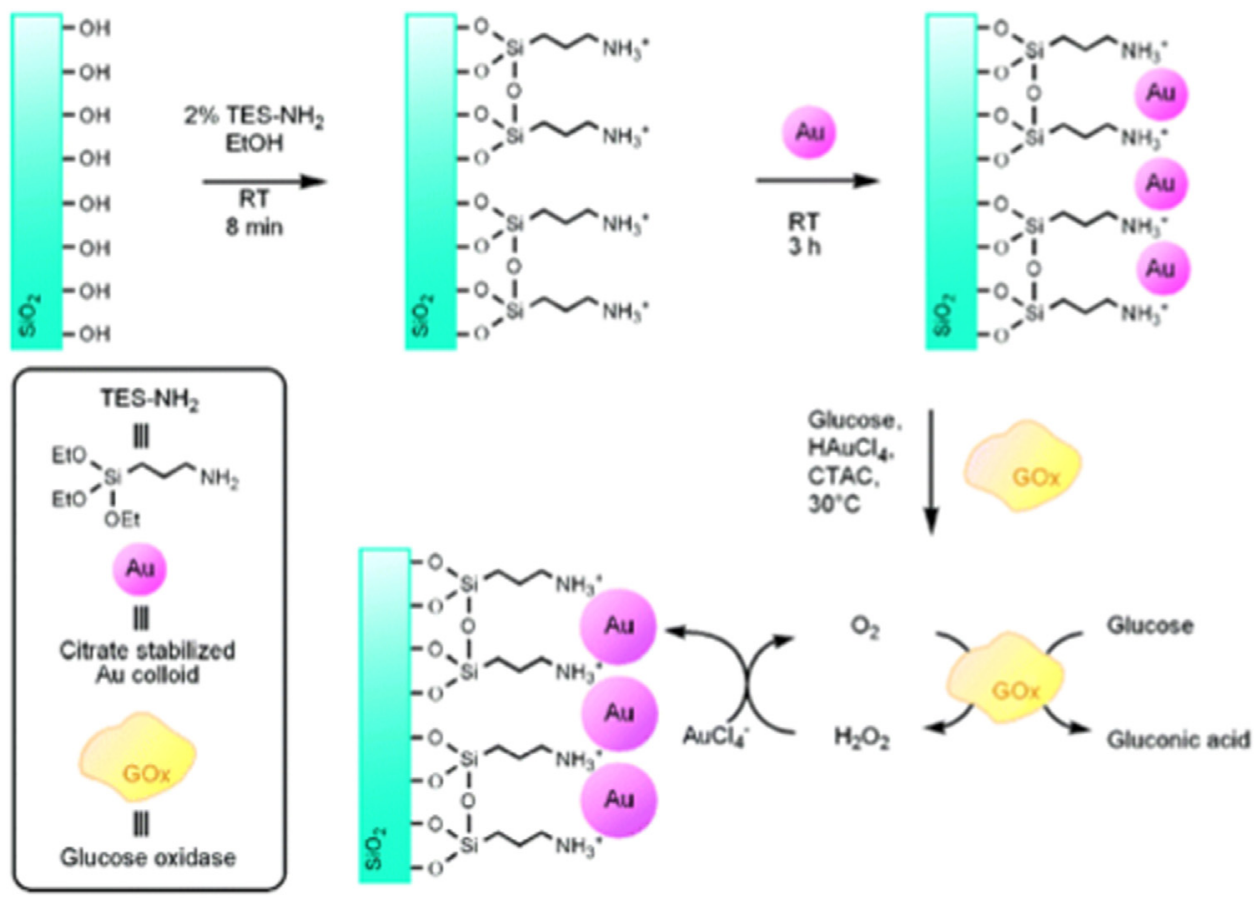

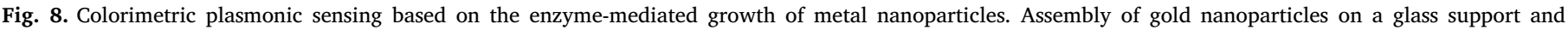

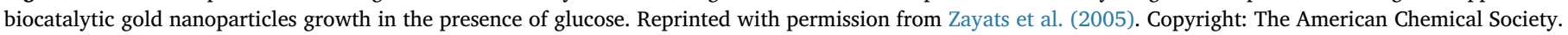
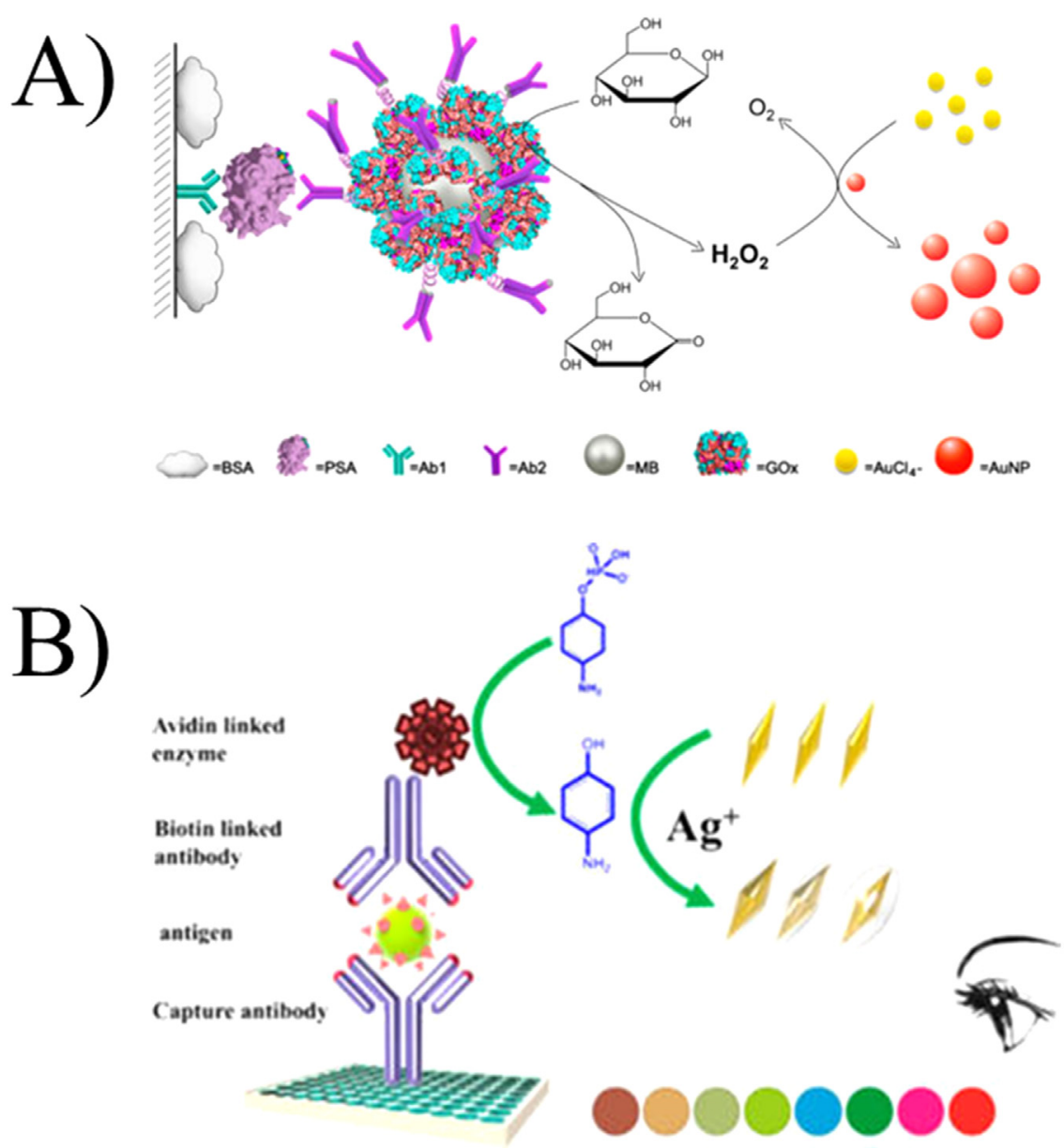

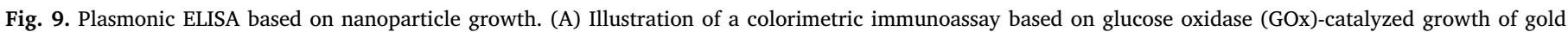

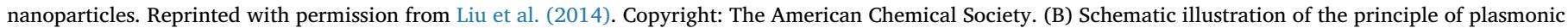

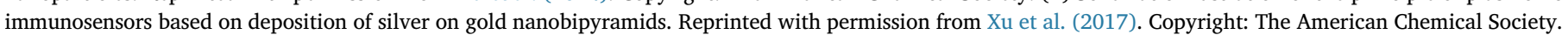



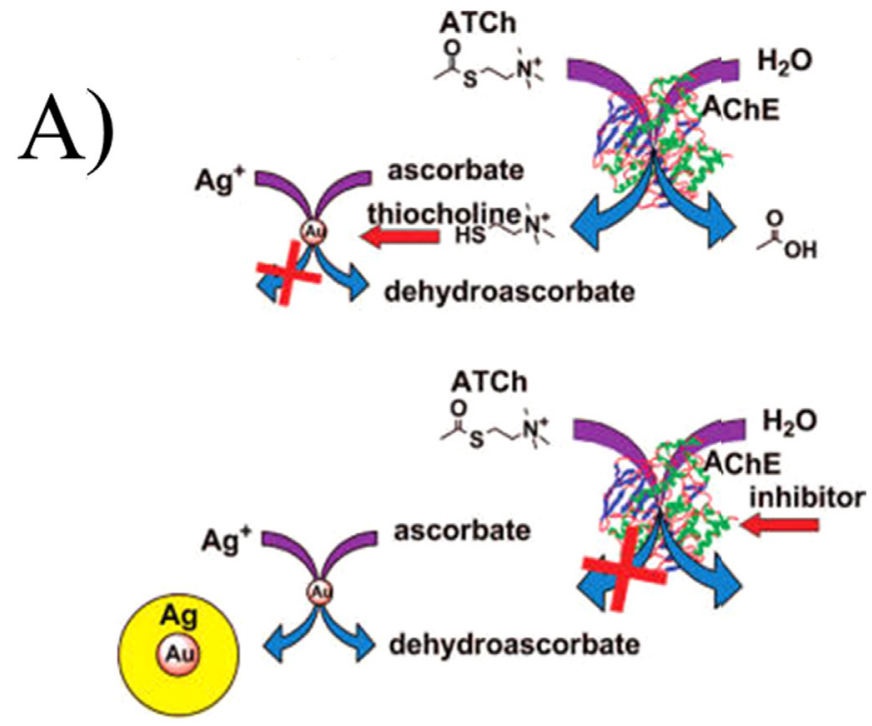

B)

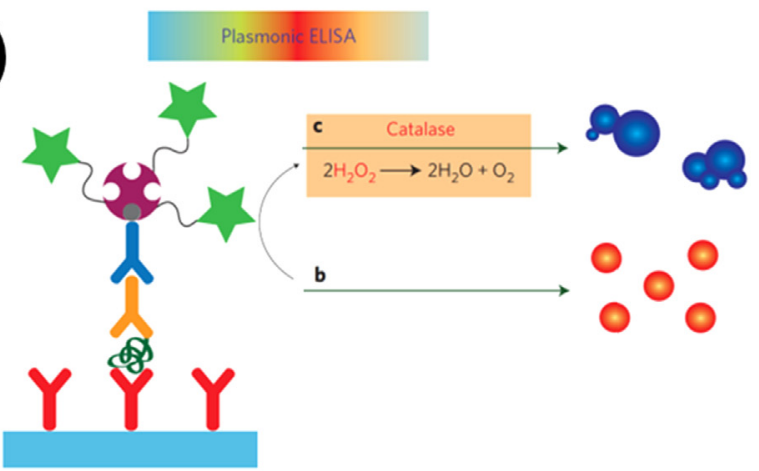

Fig. 10. Colorimetric plasmonic sensing based on inhibitor-mediated growing of metal nanoparticles. (A) Detection of acetylcholine esterase inhibitors by growing silver-coated gold nanoparticles. Reprinted with permission from Virel et al. (2008). Copyright: The American Chemical Society. (B) Schematic illustration of signal generation mechanisms for the plasmonic sandwich ELISA format based on $\mathrm{H}_{2} \mathrm{O}_{2}$-mediated formation of gold nanoparticles. Reproduced with permission from De La Rica and Stevens (2012). Copyright: Nature Publishing Group.

the LSPR band due to the growth of core/shell nanoparticles was used for quantitation of the inhibitor. Passivation during nanoparticle growth has also been used in plasmonic ELISA. For example, the Stevens group developed an ultrasensitive plasmonic ELISA assay based on controlling the growth of gold nanoparticles by enzymatic decomposition of the reducing agent $\left(\mathrm{H}_{2} \mathrm{O}_{2}\right)$ (Fig. 10B) (De La Rica and Stevens, 2012). Compared with conventional ELISA, the plasmonic ELISA used gold nanoparticles as the readout to improve sensitivity. Once the sandwich-type immunocomplex was formed in the presence of antigen, catalase binding to the secondary antibodies hydrolyzed $\mathrm{H}_{2} \mathrm{O}_{2}$ into $\mathrm{O}_{2}$, thereby decreasing the $\mathrm{H}_{2} \mathrm{O}_{2}$ concentration. As a result, the reduction of gold ions slowed, and gold nanoparticles formed, accompanied by a change in solution color to blue. In the absence of antigen, $\mathrm{H}_{2} \mathrm{O}_{2}$ concentration remained high, and gold ions were reduced rapidly, resulting in spherical dispersed gold nanoparticles and a red-colored solution. This plasmonic ELISA was able to detect two target proteins (PSA and HIV-1 capsid antigen p24) at the ultralow concentration of $10^{-18}$ $\mathrm{g} \mathrm{mL}^{-1}$ in whole serum.

\subsection{Colorimetric test strips using etching and growth of noble metal nanoparticles}

As mentioned previously, one of the advantages of "non-aggregation" plasmonic sensors is that they can be made into test strips using immobilized nanoparticles. Test strips can increase the stability of nanoparticles and prevent their aggregation. Development of test strips derived from "non-aggregation" plasmonic sensors will significantly expand the practical applications of plasmonic sensors. To date, different loading materials have successfully been used to prepare test strips; examples include filter paper, polymer brushes, agarose gel, nitrocellulose membranes, silica gel, mixed cellulose ester filter paper, and glass substrates. These test strips have been used to detect various targets including $\mathrm{Cu}^{2+}$, (Zhang et al., 2014b) $\mathrm{CN}^{-}$, (Zeng et al., 2014) $\mathrm{Pb}^{2+}$, (Ferhan et al., 2013; Lee and Huang, 2011) and dihydronicotinamide adenine dinucleotide (NADH). (Liang et al., 2015b) It is worth noting that most of these are etching-based plasmonic sensors because etching of nanoparticles is easy to control on solid surfaces.

The Chen group designed a colorimetric method for the detection of copper ions based on the etching of gold nanorods and then made test strips by immobilizing gold nanorods on filter paper (Fig. 11A). (Zhang et al., 2014b) The visual detection limit of the test paper was $100 \mathrm{nmol} /$ L. As another example, Zeng and coworkers developed a plasmonic sensor for the detection of $\mathrm{CN}^{-}$based on the etching of $\mathrm{Au} @ \mathrm{Ag}$ nanoparticles. They used agarose gel as the loading material to make test strips for the detection of $\mathrm{CN}^{-}$(Fig. 11B). (Zeng et al., 2014) They further used these test strips to analyze tap, sea, lake, and industrial water samples. The agarose gels exhibited similar color responses to ultrapure and other natural water samples. Notably, all assays were carried out using the original samples without any pretreatment steps. These results demonstrated that the test strips did not suffer from matrix effects. Similarly, the Kim group and Huang group developed $\mathrm{Pb}^{2+}$ test strips using polymer brush-loaded gold nanostructures and nitrocellulose membranes, respectively (Fig. 11C and D) (Ferhan et al., 2013; Lee and Huang, 2011).

Growth-based plasmonic sensors can also be made in the form of test strips. Compared with etching-based plasmonic sensors, these types of sensors are more difficult to control on substrate. Therefore, fewer of these types of test strips have been developed. Willner's group developed an acetylcholine esterase (AChE) assay based on the catalytic growth of Au NP seeds and applied it to quantify the presence of AChE inhibitors using colorimetric detection (Pavlov et al., 2005). AChE catalyzed the hydrolysis of acetylthiocholine to yield the reducing agent thiocholine, which promoted the growth of Au NP seeds in the presence of $\mathrm{AuCl}_{4}{ }^{-}$. In the presence of AChE inhibitors, the catalytic growth of the $\mathrm{Au}$ NPs decreased due to inhibition of AChE activity. Therefore, the strips exhibited different colors according to presence/absence of inhibitors, which could be distinguished by the naked eye (Fig. 11E).

\section{Conclusions and perspectives}

In this review, we summarized "non-aggregation" plasmonic colorimetric sensors that have been successfully used for biological and environmental analyses. Compared with "aggregation" plasmonic assays, these types of sensors are a relatively new research area. Based on the triggering of etching or growth of plasmonic nanoparticles, different targets, including metal ions, anions, DNA, and proteins, have been successfully detected with the naked eye. These "non-aggregation" plasmonic colorimetric sensors have several advantages. First, the nanoparticles used in these types of sensors are normally label-free, which avoids complex and time-consuming labeling procedures. Second, these sensors do not yield false positive results caused by auto-aggregation of nanoparticles (some of them can be used for seawater samples (Zhang et al., 2014b)). Third, "non-aggregation" plasmonic sensors can be made into practical test strips by immobilization of nanoparticles on appropriate substrates. These merits can significantly improve 


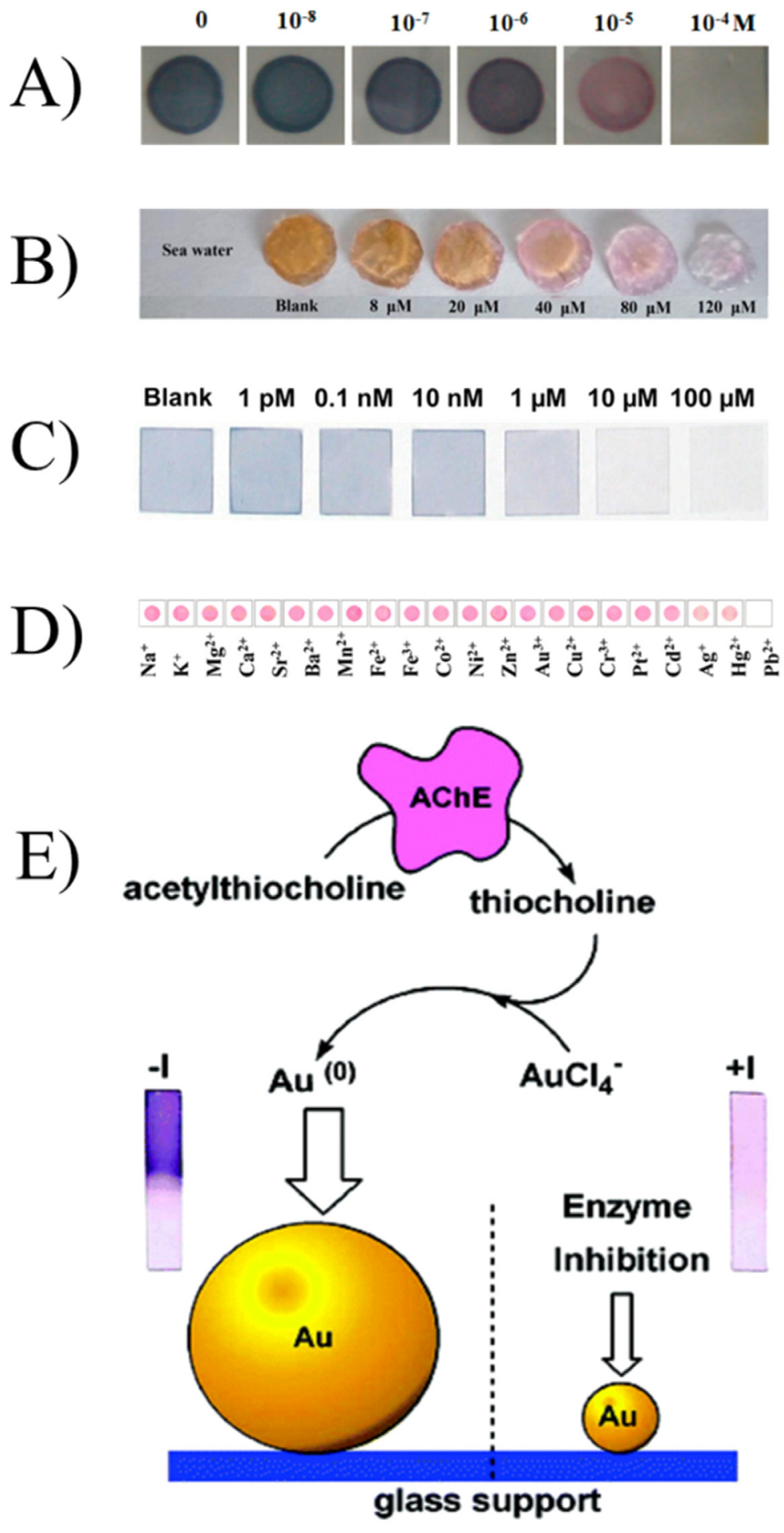

$\mathrm{Cu}^{2+}$

$\mathrm{CN}^{-}$

$\mathbf{P b}^{2+}$

$\mathbf{P b}^{2+}$
Fig. 11. Colorimetric test strips based on "non-aggregation" plasmonic colorimetric sensors. (A) Visual detection of $\mathrm{Cu}^{2+}$ ions using immobilized gold nanorods on filter paper. Reproduced with permission from Zhang et al. (2014b). Copyright: The American Chemical Society. (B) Agarose gel as the loading material to make test strips for the detection of $\mathrm{CN}^{-}$. Reproduced with permission from Zeng et al. (2014). Copyright: The Royal Society of Chemistry. (C) Polymer brush loaded with gold nanostructures for colorimetric detection of $\mathrm{Pb}^{2+}$ ions. Reproduced with permission from Ferhan et al. (2013). Copyright: The American Chemical Society. (D) Photo showing the selectivity of a $\mathrm{Pb}^{2+}$ sensor based on nitrocellulose membrane loaded with gold nanoparticles. Reproduced with permission from Lee and Huang (2011). Copyright: The American Chemical Society. (E) Scheme for detection of acetylcholine esterase inhibitors based on the mediation of the growth of silver-coated gold nanoparticles. Reproduced with permission from Pavlov et al. (2005). Copyright: The American Chemical Society. detection accuracy and reproducibility and allow detection of targets in complex samples (e.g., sea water (Zeng et al., 2014; Zhang et al., 2014b) and serum (Zhang et al., 2015b)).

However, some critical issues still need to be resolved for broader application of these sensors. Furthermore, a combination of these sensors with other types of sensing systems will significantly promote future developments in this field. Firstly, current "non-aggregation" plasmonic colorimetric sensors have been applied to a limited target range compared to "aggregation" plasmonic colorimetric sensors. The targets of "non-aggregation" plasmonic colirimetric sensors, therefore, need to be expanded. Indirect triggering of etching or growth of nanoparticles holds promise for expanding the applications of "non- aggregation" plasmonic sensors (Zhang et al., 2014a). Development of plasmonic ELISAs (De La Rica and Stevens, 2012; Zhang et al., 2015b) will also be key to mainstream application of these sensors. Additionally, the most challenging issue is to obtain standard analytical materials, namely nanoparticles which must have stable optical and chemical properties with reproducibility of synthesis. However, the nanoparticles currently available do not meet these requirements. So, more effort needs to be invested in nanoparticle preparation. Now, nanoparticles are usually obtained by chemical synthesis, but physical methods (e.g., electron beam lithography) can potentially be used to produce nanoparticles in the future. Finally, many of the methods require high incubation temperature or long incubation time. This will 
greatly limit the practical applications for the on-site detection. One of the future directions in this field is to develop "non-aggregation" plasmonic colorimetric sensors with quick response (several mins) and working condition at room temperature

"Non-aggregation" plasmonic colorimetric sensors can be improved by combining with some new technologies. (1) Paper-based microfluidic devices (Dungchai et al., 2010) may be combined with non-aggregation based plasmonic colorimetric sensors. Paper-based microfluidics has been studied intensively in the field of analytical chemistry, and the combination of paper-based microfluidics with "non-aggregation" plasmonic sensors will potentially allow the development of novel point-of-care devices. (2) A smartphone is an attractive and cheap alternative in comparison to bulky optical instrumentation due to the advantages of the built-in camera, screen/flash, and the connection to data storage (Shen et al., 2012; Wang et al., 2016). The incorporation of a colorimetric sensing system into a smartphone platform is a potentially promising way to enable the smartphone to sense, transduce and analyze on-line detection information (Shen et al., 2012), So, the integration of smartphone-based technique will be another way to push "non-aggregation" colorimetric sensors forward. (3) Multiple modes detection techniques for the same sample will improve detection accuracy and reliability. Although colorimetric plasmonic sensors are extremely useful tools for on-site detection, they usually only allow semi-quantitative determination of the target. The fluorescence of quantum dots or organic molecules will be quenched when they are close to the plasmonic nanoparticles. It has been reported that the fluorescence of corban nanomaterials can be recovered when the adjacent plasmonic structures are etched by targets (Fu et al., 2012; Ran et al., 2013). So, it is possible to integrate fluorescent methods with etching-based plasmonic colorimetric sensors. Surface enhanced Raman scattering (SERS) is sensitive to the size and shape of plasmonic structures (Boyack and Le Ru, 2009). Integration of "non-aggregation" plasmonic sensors with SERS might facilitate the development of novel, extremely sensitive detection devices. Also, circular dichroism (CD ) spectroscopy of plasmonic structures is also sensitive to the shape (Li et al., 2012), composition (Wu et al., 2015b), and symmetry of nanostructures, so incorporation of plasmonic CD spectroscopy with "nonaggregation" plasmonic sensors may also be possible.

Advances in material science and new analytical techniques will enhance the potential applications of metal nanoparticles etching- or growth-based "non-aggregation" plasmonic sensors.

\section{Acknowledgements}

This work was supported by grants from the National Nature Science Foundation of China (grant numbers: 21575159, 21477160, 81573393, 21275158), the National Research Foundation of Korea (grant numbers: 2008-0061891, 2009-00426), and the Korea Health Industry Development Institute (grant number: HI16C2129).

\section{References}

Baptista, P., Pereira, E., Eaton, P., Doria, G., Miranda, A., Gomes, I., Quaresma, P., Franco, R., 2008. Anal. Bioanal. Chem. 391, 943-950.

Baron, R., Zayats, M., Willner, I., 2005. Anal. Chem. 77, 1566-1571.

Boyack, R., Le Ru, E.C., 2009. Phys. Chem. Chem. Phys. 11, 7398-7405.

Cecchin, D., De La Rica, R., Bain, R., Finnis, M.W., Stevens, M., Battaglia, G., 2014. Nanoscale 6, 9559-9562.

Chang, C.-C., Wang, G., Takarada, T., Maeda, M., 2017. ACS Appl. Mater. Interfaces 9, 34518-34525.

Chemnasiri, W., Hernandez, F.E., 2012. Sens. Actuators B 173, 322-328.

Chen, L., Fu, X., Lu, W., Chen, L., 2012a. ACS Appl. Mater. Interfaces 5, 284-290.

Chen, H., Shao, L., Li, Q., Wang, J., 2013a. Chem. Soc. Rev. 42, 2679-2724.

Chen, J., Jackson, A.A., Rotello, V.M., Nugen, S.R., 2016a. Small 12, 2469-2475.

Chen, L., Lou, T., Yu, C., Kang, Q., Chen, L., 2011. Analyst 136, 4770-4773.

Chen, N., Zhang, Y., Liu, H., Ruan, H., Dong, C., Shen, Z., Wu, A., 2016a. ACS Sustain. Chem. Eng. 4, 6509-6516.

Chen, N., Zhang, Y., Liu, H., Wu, X., Li, Y., Miao, L., Shen, Z., Wu, A., 2016b. ACS Sensors 1. pp. 521-527.

Chen, Y.-Y., Chang, H.-T., Shiang, Y.-C., Hung, Y.-L., Chiang, C.-K., Huang, C.-C., 2009.
Anal. Chem. 81, 9433-9439.

Chen, Z., Liu, R., Wang, S., Qu, C., Chen, L., Wang, Z., 2013b. RSC Adv. 3, 13318-13323.

Chen, Z., Zhang, C., Wu, Q., Li, K., Tan, L., 2015. Sens. Actuators, B 220, 314-317.

Chen, Z., Zhang, Z., Qu, C., Pan, D., Chen, L., 2012 b. Analyst 137, 5197-5200.

Cheng, F., Chen, Z., Zhang, Z., Chen, L., 2016. Analyst 141, 1918-1921.

De La Rica, R., Stevens, M.M., 2012. Nat. Nanotechnol. 7, 821-824.

De La Rica, R., Stevens, M.M., 2013. Nat. Protoc. 8, 1759-1764.

Dungchai, W., Chailapakul, O., Henry, C.S., 2010. Anal. Chim. Acta 674, 227-233.

Elghanian, R., Storhoff, J.J., Mucic, R.C., Letsinger, R.L., Mirkin, C.A., 1997. Science 277, 1078-1081.

Fang, X., Ren, H., Zhao, H., Li, Z., 2017. Microchim. Acta 184, 415-421.

Ferhan, A.R., Guo, L., Zhou, X., Chen, P., Hong, S., Kim, D.-H., 2013. Anal. Chem. 85, 4094-4099.

Fu, X., Lou, T., Chen, Z., Lin, M., Feng, W., Chen, L., 2012. ACS Appl. Mater. Interfaces 4, 1080-1086.

Gao, Z., Deng, K., Wang, X.-D., Miró, M., Tang, D., 2014. ACS Appl. Mater. Interfaces 6 , $18243-18250$.

Ghosh, S.K., Pal, T., 2007. Chem. Rev. 107, 4797-4862.

Gu, Y., Kong, S., Diao, X., Guo, Y., Zhang, K., He, H., 2016. New J. Chem. 40, 7557-7563.

Guo, L., Chen, L., Hong, S., Kim, D.-H., 2016a. Biosens. Bioelectron. 79, 266-272.

Guo, L., Jackman, J.A., Yang, H.-H., Chen, P., Cho, N.-J., Kim, D.-H., 2015. Nano Today $10,213-239$.

Guo, L., Xu, S., Ma, X., Qiu, B., Lin, Z., Chen, G., 2016b. Sci. Rep. 6, 32755.

Guo, Y., Wu, J., Li, J., Ju, H., 2016c. Biosens. Bioelectron. 78, 267-273.

He, Y., Yu, H., 2015. Analyst 140, 902-906.

Huang, X., Chen, R., Xu, H., Lai, W., Xiong, Y., 2016. Anal. Chem. 88, 1951-1958.

Jana, N.R., Gearheart, L., Murphy, C.J., 2001. Langmuir 17, 6782-6786.

Jang, H., Min, D.-H., 2015. RSC Adv. 5, 14330-14332.

Jin, L.-H., Han, C.-S., 2014. Sens. Actuators B 195, 239-245.

Kaushik, A., Kumar, R., Arya, S.K., Nair, M., Malhotra, B., Bhansali, S., 2015. Chem. Rev. 115, 4571-4606.

Kelly, K.L., Coronado, E., Zhao, L.L., Schatz, G.C., 2003a. J. Phys. Chem. B 107, 668-677.

Kelly, K.L., Coronado, E., Zhao, L.L., Schatz, G.C., 2003b. The optical properties of meta nanoparticles: the influence of size, shape, and dielectric environment. J. Phys. Chem. B 668-677.

Kim, F., Song, J.H., Yang, P., 2002. J. Am. Chem. Soc. 124, 14316-14317.

Lan, Y.-J., Lin, Y.-W., 2014. Anal. Methods 6, 7234-7242.

Lee, J.S., Han, M.S., Mirkin, C.A., 2007. Angew, Chem. 119, 4171-4174.

Lee, K.-S., El-Sayed, M.A., 2005. J. Phys. Chem. B 109, 20331-20338.

Lee, S., Nam, Y.-S., Choi, S.-H., Lee, Y., Lee, K.-B., 2016. Microchim. Acta 183, 3035-3041.

Lee, Y.-F., Huang, C.-C., 2011. ACS Appl. Mater. Interfaces 3, 2747-2754.

Li, F.-M., Liu, J.-M., Wang, X.-X., Lin, L.-P., Cai, W.-L., Lin, X., Zeng, Y.-N., Li, Z.-M., Lin, S.-Q., 2011. Sens. Actuators B 155, 817-822.

Li, T., Li, Y., Zhang, Y., Dong, C., Shen, Z., Wu, A., 2015. Analyst 140, 1076-1081.

Li, Y., Li, Z., Gao, Y., Gong, A., Zhang, Y., Hosmane, N.S., Shen, Z., Wu, A., 2014. Nanoscale 6, 10631-10637.

Li, Y., Ma, X., Xu, Z., Liu, M., Lin, Z., Qiu, B., Guo, L., Chen, G., 2016. Analyst 141 2970-2976.

Li, Z., Zhu, Z., Liu, W., Zhou, Y., Han, B., Gao, Y., Tang, Z., 2012. J. Am. Chem. Soc. 134, $3322-3325$.

Liang, J., Yao, C., Li, X., Wu, Z., Huang, C., Fu, Q., Lan, C., Cao, D., Tang, Y., 2015a. Biosens. Bioelectron. 69, 128-134.

Liang, P., Yu, H., Guntupalli, B., Xiao, Y., 2015b. ACS Appl. Mater. Interfaces 7, 15023-15030.

Lin, Y., Zhao, M., Guo, Y., Ma, X., Luo, F., Guo, L., Qiu, B., Chen, G., Lin, Z., 2016. Sci. Rep. 6, 37879.

Link, S., El-Sayed, M., 2005. J. Phys. Chem. B 109, 10531-10532.

Liu, D., Yang, J., Wang, H.-F., Wang, Z., Huang, X., Wang, Z., Niu, G., Hight Walker, A., Chen, X., 2014. Anal. Chem. 86, 5800-5806.

Liu, J.-M., Jiao, L., Cui, M.-L., Lin, L.-P., Wang, X.-X., Zheng, Z.-Y., Zhang, L.-H., Jiang, S. L., 2013a. Sens. Actuators B 188, 644-650.

Liu, J.-M., Wang, X.-X., Cui, M.-L., Lin, L.-P., Jiang, S.-L., Jiao, L., Zhang, L.-H., 2013b. Sens. Actuators B 176, 97-102.

Liu, J., Lu, Y., 2003. J. Am. Chem. Soc. 125, 6642-6643.

Liu, M., Lin, Z, Lin, J.-M., 2010. Anal. Chim. Acta 670, 1-10.

Liu, R., Chen, Z., Wang, S., Qu, C., Chen, L., Wang, Z., 2013c. Talanta 112, 37-42.

Liu, X., Zhang, S., Tan, P., Zhou, J., Huang, Y., Nie, Z., Yao, S., 2013d. Chem. Commun. 49, 1856-1858.

Liu, Y., Lv, B., Liu, A., Liang, G., Yin, L., Pu, Y., Wei, W., Gou, S., Liu, S., 2018. Sens. Actuators B.

Liz-Marzán, L.M., 2004. Mater. Today 7, 26-31.

Lu, S., Chen, L., Yang, P., Matras-Postolek, K., 2016. RSC Adv, 6, 19620-19625.

Ma, X., Chen, Z., Kannan, P., Lin, Z., Qiu, B., Guo, L., 2016. Anal. Chem. 88, 3227-3234.

Ma, X., Lin, Y., Guo, L., Qiu, B., Chen, G., Yang, H.-h., Lin, Z., 2017. Biosens. Bioelectron. $87,122-128$

Malile, B., Chen, J.I., 2013. J. Am. Chem. Soc. 135, 16042-16045.

Mayer, K.M., Hafner, J.H., 2011. Chem. Rev. 111, 3828-3857.

Nehl, C.L., Hafner, J.H. 2008, J. Mater. Chem. 18, 2415-2419.

Oh, J.-H., Kim, B.C., Lee, J.-S., 2014. Anal. Bioanal. Chem. 406, 7591-7600.

Okuno, Y., Nishioka, K., Kiya, A., Nakashima, N., Ishibashi, A., Niidome, Y., 2010. Nanoscale 2, 1489-1493.

Pavlov, V., Xiao, Y., Willner, I., 2005. Nano Lett. 5, 649-653.

Peng, M.-P., Ma, W., Long, Y.-T., 2015. Anal. Chem. 87, 5891-5896.

Ran, X., Sun, H., Pu, F., Ren, J., Qu, X., 2013. Chem. Commun. 49, 1079-1081.

Rex, M., Hernandez, F.E., Campiglia, A.D., 2006. Anal. Chem. 78, 445-451. 
Saa, L., Coronado-Puchau, M., Pavlov, V., Liz-Marzán, L.M., 2014. Nanoscale 6, 7405-7409.

Saa, L., Grinyte, R., Sánchez-Iglesias, A., Liz-Marzán, L.M., Pavlov, V., 2016. ACS Appl. Mater. Interfaces 8, 11139-11146.

Saha, K., Agasti, S.S., Kim, C., Li, X., Rotello, V.M., 2012. Chem. Rev. 112, 2739-2779. Sasikumar, T., Ilanchelian, M., 2017. Anal. Methods.

Shen, L., Hagen, J.A., Papautsky, I., 2012. Lab Chip 12, 4240-4243.

Siegel, G., Roedel, H., Nolte, J., Hofer, H., Bertsche, O., 1976. Physiol. Smooth Muscle 19-39.

Soh, J.H., Lin, Y., Rana, S., Ying, J.Y., Stevens, M.M., 2015. Anal. Chem. 87, 7644-7652. Talley, C.E., Jackson, J.B., Oubre, C., Grady, N.K., Hollars, C.W., Lane, S.M., Huser, T.R., Nordlander, P., Halas, N.J., 2005. Nano Lett. 5, 1569-1574.

Tan, K., Yang, G., Chen, H., Shen, P., Huang, Y., Xia, Y., 2014. Biosens. Bioelectron. 59, 227-232.

Tang, L., Li, J., 2017. ACS Sensors 2. pp. 857-875.

Tang, Y., Zhang, W., Liu, J., Zhang, L., Huang, W., Huo, F., Tian, D., 2015. Nanoscale 7, 6039-6044.

Tripathy, S.K., Woo, J.Y., Han, C.-S., 2011. Anal. Chem. 83, 9206-9212.

Vilela, D., González, M.C., Escarpa, A., 2012. Anal. Chim. Acta 751, 24-43.

Virel, A., Saa, L., Pavlov, V., 2008. Anal. Chem. 81, 268-272.

Wang, G., Chen, Z., Chen, L., 2011. Nanoscale 3, 1756-1759.

Wang, S., Bi, S., Wang, Z., Xia, J., Zhang, F., Yang, M., Gui, R., Li, Y., Xia, Y., 2015. Chem. Commun. 51, 7927-7930.

Wang, S., Chen, Z., Chen, L., Liu, R., Chen, L., 2013a. Analyst 138, 2080-2084.

Wang, X.-X., Liu, J.-M., Jiang, S.-L., Jiao, L., Lin, L.-P., Cui, M.-L., Zhang, X.-Y., Zhang, L.H., Zheng, Z.-Y., 2013b. Sens. Actuators, B 182, 205-210.

Wang, X., Chen, L., Chen, L., 2014. Microchim. Acta 181, 105-110.

Wang, Y., Liu, X., Chen, P., Tran, N.T., Zhang, J., Chia, W.S., Boujday, S., Liedberg, B., 2016. Analyst 141, 3233-3238.

Wu, D., Lu, H.-F., Xie, H., Wu, J., Wang, C.-M., Zhang, Q.-L., 2015a. Sens. Actuators, B 221, 1433-1440.

Wu, S., Li, D., Gao, Z., Wang, J., 2017. Microchim. Acta 184, 4383-4391.

Wu, X., Xu, L., Ma, W., Liu, L., Kuang, H., Yan, W., Wang, L., Xu, C., 2015b. Adv. Funct. Mater. 25, 850-854.

Xia, Y., Ye, J., Tan, K., Wang, J., Yang, G., 2013. Anal. Chem. 85, 6241-6247.

Xianyu, Y., Sun, J., Li, Y., Tian, Y., Wang, Z., Jiang, X., 2013. Nanoscale 5, 6303-6306.

Xin, J., Zhang, F., Gao, Y., Feng, Y., Chen, S., Wu, A., 2012. Talanta 101, 122-127.

Xiong, Y., Zhang, Y., Rong, P., Yang, J., Wang, W., Liu, D., 2015. Nanoscale 7,
15584-15588.

Xu, S., Ouyang, W., Xie, P., Lin, Y., Qiu, B., Lin, Z., Chen, G., Guo, L., 2017. Anal. Chem. 89, 1617-1623.

Xuan, Z., Li, M., Rong, P., Wang, W., Li, Y., Liu, D., 2016. Nanoscale 8, 17271-17277. Yan, J., Wang, L., Tang, L., Lin, L., Liu, Y., Li, J., 2015. Biosens. Bioelectron. 70, 404-410.

Yang, H., Liu, A., Wei, M., Liu, Y., Lv, B., Wei, W., Zhang, Y., Liu, S., 2017. Anal. Chem. 89, 12094-12100.

Yang, R., Song, D., Wang, C., Zhu, A., Xiao, R., Liu, J., Long, F., 2015. RSC Adv. 5, 102542-102549.

Yang, X., Gao, Z., 2015. Chem. Commun. 51, 6928-6931.

Yang, X., Yu, Y., Gao, Z., 2014. ACS Nano 8, 4902-4907.

Yao, C., Yu, S., Li, X., Wu, Z., Liang, J., Fu, Q., Xiao, W., Jiang, T., Tang, Y., 2017. Anal. Bioanal. Chem. 409, 1093-1100.

Yu, F., Li, Y., Li, M., Tang, L., He, J.-J., 2017. Biosens. Bioelectron. 89, 880-885.

Zayats, M., Baron, R., Popov, I., Willner, I., 2005. Nano Lett. 5, 21-25.

Zeng, J.-b., Cao, Y.-y., Chen, J.-j., Wang, X.-d., Yu, J.-f., Yu, B.-b., Yan, Z.-f., Chen, X., 2014. Nanoscale 6, 9939-9943.

Zhang, Z., Chen, Z., Chen, L., 2015a. Langmuir 31, 9253-9259.

Zhang, Z., Chen, Z., Cheng, F., Zhang, Y., Chen, L., 2016. Analyst 141, 2955-2961.

Zhang, Z., Chen, Z., Cheng, F., Zhang, Y., Chen, L., 2017. Biosens. Bioelectron. 89, 932-936.

Zhang, Z., Chen, Z., Pan, D., Chen, L., 2014a. Langmuir 31, 643-650.

Zhang, Z., Chen, Z., Qu, C., Chen, L., 2014b. Langmuir 30, 3625-3630.

Zhang, Z., Chen, Z., Wang, S., Cheng, F., Chen, L., 2015b. ACS Appl. Mater. Interfaces 7. 27639-27645.

Zhao, J., Zhang, X., Yonzon, C.R., Haes, A.J., Van Duyne, R.P., 2006. Nanomedicine 1, 219-228.

Zhao, L., Wiebe, J., Zahoor, R., Slavkovic, S., Malile, B., Johnson, P.E., Chen, J.I., 2016. Anal. Methods 8, 6625-6630.

Zhong, Z., Patskovskyy, S., Bouvrette, P., Luong, J.H., Gedanken, A., 2004. J. Phys. Chem. B 108, 4046-4052.

Zhou, C.-H., Zhao, J.-Y., Pang, D.-W., Zhang, Z.-L., 2014. Anal. Chem. 86, 2752-2759.

Zhou, D., Xie, G., Cao, X., Chen, X., Zhang, X., Chen, H., 2016. Microchim. Acta 183, 2753-2760.

Zhou, W., Gao, X., Liu, D., Chen, X., 2015a. Chem. Rev. 115, 10575-10636.

Zhou, W., Gao, X., Liu, D., Chen, X., 2015b. Chem. Rev. 115, 10575-10636.

Zhu, J., Yu, Y.-Q., Li, J.-J., Zhao, J.-W., 2016. RSC Adv. 6, 25611-25619. 